\title{
Integrated cell-free DNA and cytokine analysis uncovers distinct tissue injury and immune response patterns in solid organ transplant recipients with COVID-19
}

\author{
Temesgen Andargie \\ National Institutes of Health \\ Weiqiang Zhou \\ Johns Hopkins University \\ Andrew Karaba \\ Johns Hopkins University \\ Taibo Li
}

Johns Hopkins University https://orcid.org/0000-0002-6624-9293

Fayaz Seifuddin

National Institutes of Health

Alex Rittenhouse

Johns Hopkins University

Hyesik Kong

National Institutes of Health

Komudi Singh

National Heart Lung and Blood Institute https://orcid.org/0000-0002-6413-541X

Robert Woodward

CareDx, Inc.

Aldo lacono

University of Maryland

Robin Avery

Johns Hopkins University

Mehdi Pirooznia

National Institutes of Health https://orcid.org/0000-0002-4210-6458

Moon Jang

National Institutes of Health

Hongkai Ji

Johns Hopkins Bloomberg School of Public Health https://orcid.org/0000-0002-6480-0141

Andrea Cox

Johns Hopkins University 
Sean Agbor-Enoh ( $\square$ sean.agbor-enoh@nih.gov )

National Institutes of Health https://orcid.org/0000-0002-9380-9161

Article

Keywords:

Posted Date: January 20th, 2022

DOI: https://doi.org/10.21203/rs.3.rs-1262270/v1

License: (c) (1) This work is licensed under a Creative Commons Attribution 4.0 International License. Read Full License 
1 Integrated cell-free DNA and cytokine analysis uncovers distinct tissue injury and immune

2 response patterns in solid organ transplant recipients with COVID-19

3 Temesgen E. Andargie ${ }^{1,2}$, Weiqiang Zhou ${ }^{3}$, Andrew H. Karaba ${ }^{4}$, Taibo Li ${ }^{3}$, Fayaz Seifuddin ${ }^{5}$,

4 Alex G. Rittenhouse ${ }^{3}$, Hyesik Kong ${ }^{1}$, Komudi Singh ${ }^{5}$, Robert Woodward ${ }^{6}$, Aldo lacono ${ }^{7}$,

5 Robin K Avery ${ }^{4}$, Mehdi Pirooznia ${ }^{5}$, Moon Kyoo Jang ${ }^{1}$, Hongkai $\mathrm{Ji}^{3}$, Andrea L. Cox ${ }^{4}$, Sean

6 Agbor-Enoh ${ }^{*}, 1,4$

71 Genomic Research Alliance for Transplantation (GRAfT) and Laboratory of Applied

8 Precision Omics, National Heart, Lung, and Blood Institute (NHLBI), NIH, Bethesda, MD.

92 Department of Biology, Howard University, Washington DC. 3 Department of

10 Biostatistics, Bloomberg School of Public Health, Johns Hopkins University, Baltimore,

11 MD. 4 Department of Medicine, Johns Hopkins University, School of Medicine, Baltimore,

12 MD. 5 Bioinformatics and Computation Core, NHLBI, MD. 6 CareDx, Brisbane, CA. 7

13 Department of Medicine, University of Maryland, College Park, MD.

14 *Address Correspondence to:

15 Sean Agbor-Enoh, MD, PhD

16 Lasker Clinical Research Tenure Track Investigator

17 Laboratory of Applied Precision Omics

18 Genomic Research Alliance for Transplantation (GRAfT)

19 Division of Intramural Research

20 National Heart, Lung and Blood Institute

2110 Center Dr., Rm 7D5, Bethesda, MD 20892

22 sean.agbor-enoh@nih.gov 


\section{Abstract}

2 COVID-19 pathogenesis is associated with an exuberant inflammatory response.

3 However, the tissue injury pattern and immune response in solid-organ transplant

4 recipients (SOTRs) taking immunosuppressive therapy have not been well characterized.

5 Here, we perform both cfDNA and cytokine profiling on plasma samples to map tissue

6 damage, including allograft injury and delineate underlying immunopathology. We

7 identified injuries from multiple-tissue types, including hematopoietic cells, vascular

8 endothelium, hepatocyte, adipocyte, pancreas, kidney, heart, and lung in SOTRs with

9 COVID-19 that correlates with disease severity. SOTRs with COVID-19 have higher

10 plasma levels of cytokines such as IFN- $\lambda 1$, IFN- $\mathrm{y}$, IL-15, IL-18 IL-1RA, IL-6, MCP-2, and

11 TNF- $\alpha$ as compared to healthy controls, and the levels of GM-CSF, IL-15, IL-6, IL-8, and

12 IL-10 were associated with disease severity in SOTRs. Strikingly, IFN- $\lambda$ and IP-10 were

13 markedly increased in SOTRs compared to immunocompetent patients with COVID-19.

14 Correlation analyses showed a strong association between monocyte-derived cfDNA and

15 inflammatory cytokines/chemokines in SOTRs with COVID-19. Moreover, compared to

16 other respiratory viral infections, COVID-19 induced pronounced injury in hematopoietic,

17 vascular endothelial and endocrine tissues. Allograft injury, measured as donor-derived

18 cfDNA was elevated in SOTRs with COVID-19, including allografts distant from the

19 primary site of infection. Allograft injury correlated with inflammatory cytokines and cfDNA

20 from immune cells. Furthermore, longitudinal analysis identified a gradual decrease of

21 cfDNA and inflammatory cytokine levels in patients with a favorable outcome. Our findings 
1 highlight distinct tissue injury and cytokine features in SOTRs with COVID-19 that

2 correlate with disease severity. 


\section{Introduction}

2 Advances in solid organ transplantation (SOT) have resulted in improved survival in

3 patients with end-stage organ failure, and there are nearly 1.5 million people living with

4 organ transplants worldwide ${ }^{1}$. However, viral infections after SOT are a major cause of

5 severe disease and reduced allograft and patient survival ${ }^{2-4}$, due to immunosuppression

6 and a high prevalence of comorbidities. The current COVID-19 pandemic continues to

7 threaten the world with over 275 million cases and 5.3 million deaths as of December 21,

82021 (https://coronavirus.jhu.edu/). Similar concerns exist in SOT recipients (SOTRs)

9 infected with SARS-CoV-2. The clinical spectrum of COVID-19 in transplant patients has

10 been variable in published reports, ranging from mild disease to severe multiple-organ

11 failure and death ${ }^{3,5-7}$. However, the tissue source of injury that contributes to these variable

12 clinical trajectories remains poorly defined. The immunogenicity of COVID-19 vaccines

13 is suboptimal in SOTR ${ }^{8-10}$ and new SARS-CoV-2 variants continue to emerge ${ }^{11}$, which is

14 worsening the situation. It has also been shown in some studies that immunosuppressive

15 drug mycophenolate mofetil (MMF) is linked to severe COVID-19 outcome ${ }^{12}$ and to lower

16 antibody responses after mRNA vaccines in SOTRs ${ }^{13}$. It is therefore critical to develop

17 approaches that map the tissue source of injury (including allograft), the pathogenesis,

18 and predict long-term outcomes. Such approaches may guide optimal timing to initiate

19 aggressive treatment towards reducing severe disease in SOTRs.

20 Circulating cell-free DNA (cfDNA), released into the bloodstream from cell injury or death,

21 is a promising noninvasive biomarker for the diagnosis and monitoring of different disease

22 ststes ${ }^{14-18}$. Healthy individuals have small amounts of cfDNA, predominantly arising from 
1 normal cell turnover of hematopoietic cell lineages ${ }^{19}$. However, in individuals with

2 pathological conditions, the concentration of cfDNA is elevated, with a significant amount

3 of cfDNA emanating from the affected tissues ${ }^{19}$. In SOT patients, allograft injury occurs

4 resulting in elevated donor-derived cfDNA (ddcfDNA) in the recipient's circulation,

5 released from allografts. Quantifying ddcfDNA by targeting single-nucleotide

6 polymorphisms (SNPs) has become a promising clinical tool for real-time monitoring of

7 allograft health ${ }^{20,21}$. Indeed, we have demonstrated ddcfDNA as a sensitive biomarker to

8 detect allograft rejection and predict long-term outcomes ${ }^{22,23}$. In addition, cfDNA also

9 carries cell-type-specific epigenetic methylation signatures and chromatine footprints.

10 Recent studies used genome-wide DNA methylation patterns to deconvolute cfDNA

11 tissues of origin and infer cell death in specific tissue or organ types ${ }^{19,24-26}$. In the general

12 population with COVID-19 patients, we and others have reported markedly elevated

13 cfDNA levels from divergent tissue types that correlated with disease progression and

14 outcome ${ }^{27,28}$. However, the effect of SARS-CoV-2 infections on the allograft and recipient

15 tissue cell and tissue types of SOTRs with COVID-19 is not completely delineated.

16 The pathogenesis of COVID-19 is multifactorial, involving direct viral infection, a

17 hyperinflammatory response, immunothrombosis, and coagulopathy ${ }^{29}$. SARS-CoV-2 uses

18 angiotensin-converting enzyme 2 for cell entry, which is expressed in multiple tissue

19 types, including lung, heart, kidney and blood vessels ${ }^{30}$. Notably, most of the severe

20 COVID-19 complications that result in multiorgan failure and death are primarily attributed

21 to virus-independent immunopathologic mechanisms ${ }^{30}$. Indeed, high serum levels of

22 inflammatory cytokines such as interleukin-6 (IL-6), IL-8, and tumor necrosis factor- $\alpha$ 
1 (TNF- $\alpha$ ) are observed in COVID-19 patients and predicted disease severity and poor

2 outcome ${ }^{31,32}$. Additionally, cfDNA is also considered as a trigger of deleterious

3 proinflammatory and prothrombotic pathways in addition to being a biomarker ${ }^{33-36}$. In

4 SOTRs, the immunosuppressive drugs taken to prevent allograft rejection may be

5 beneficial in suppressing this hyperinflammatory state and protecting severe forms of

6 COVID-19, but this could also diminish host defense against the virus. However, studies

7 comparing the relationship between tissue injury patterns and immune response in

8 SOTRs with COVID-19 and immunocompetent hosts are scarce.

9 In this study of SOTRs with COVID-19, we provide evidence of significant tissue injury that correlated with disease severity. Using whole-genome bisulfite sequencing and a library

11 of tissue-specific DNA methylation markers, we show that COVID-19 induces injury for

12 multiple tissue types, including transplanted organs that correlated with disease severity.

13 We also show a comparable tissue injury profile between SOTRs with COVID-19 and Non-

14 SOT COVID-19 patients matched for disease severity. In addition, we characterized the

15 immune response using a multiplex cytokine assay and correlated it with cfDNA profiles.

16 SOTRs with COVID-19 have distinct cytokine signatures that are strongly correlated with

17 myeloid-derived cfDNA profiles. We further reveal COVID-19 specific tissue injury

18 characteristics in lung transplant recipients (LTRs) as compared to other respiratory viral

19 infections (other RVIs). Finally, we demonstrated that longitudinal cfDNA and

20 proinflammatory cytokine levels remained elevated in a patient who eventually died while

21 gradually decreasing in recovered patients. Taken together, these data identify distinct

22 mechanisms of pathology in SOTRs and highlight integrative analysis of cfDNA and 
1 cytokine signatures as a comprehensive molecular marker of tissue injury and 2 inflammation that may help predict disease severity in SOTRs with COVID-19. 


\section{Results}

\section{Demographics and clinical characteristics of SOT recipients with COVID-19}

A total of 44 consecutive SOTRs requiring hospitalization with confirmed COVID-19 were included: 18 (40.9\%) lung, 16 (36.4\%) kidney, 5 (11.6\%) liver, 3 (6.8\%) heart, and 1 (2.3\%) combined kidney-liver recipients (Fig.1a). The median age was 54.5 years (interquartile range [IQR]: 43.5 - 66.5), the median time from transplantation was four years (IQR: 18.5), and 28 (61.4\%) patients were male. The median body mass index (BMI) was 28.3 $\mathrm{kg} / \mathrm{m}^{2}$ (IQR: $\left.24.15-34.28\right)$. The most common comorbidities were hypertension $(n=27$, $61.4 \%)$ and diabetes $(n=21,47.7 \%)$. The median length of stay in the hospital was eight days (IQR: 6-20), and all the SOTRs were on an immunosuppressive regimen. Thirty patients $(68.2 \%)$ were on a regimen containing mycophenolate prior to COVID-19 diagnosis, and it was discontinued in the majority of the patients $(n=27 ; 90 \%)$ upon admission. All patients received tacrolimus. Among the 44 SOTRs, $12(31.5 \%)$ patients developed severe COVID-19 with ICU admission during their disease course and 32 patients had $\mathrm{mild} / \mathrm{moderate}$ disease and eventually recovered. Compared to patients with mild/moderate disease, those who developed severe disease were older (median age 62.5 vs. 50 years, $p=0.03$ ), and on admission had higher WBC count ( 7.5 vs. $4.7 \mathrm{k} / \mathrm{uL}$, $p=0.015)$, absolute neutrophil count (ANC) ( 5.9 vs. $3.1 \mathrm{k} / \mathrm{uL}, \mathrm{p}=0.002)$, and D-dimer (2.57 vs. $0.47 \mathrm{mg} / \mathrm{L}, \mathrm{p}=0.001)$ upon admission. There also had longer hospitalization stay $(26.5$ vs. 7 days, $p<0.001)$. More than half of COVID-19 patients were treated with remdesivir $(n=23,52.3 \%)$ and $45 \%$ of the patients received convalescent plasma. Three of the 12 SOTRs with severe disease died due to complications of COVID-19. Baseline 
1 demographic and clinical characteristics of the SOT patients are shown in table 1. The

2 study also included 40 Non-SOT COVID-19 patients to investigate whether the tissue

3 injury pattern is different from COVID-19 SOTRs. Non-SOT COVID-19 group was similar

4 to SOT COVID-19 regarding demographics, disease severity scores, need for ICU care

5 and survival (Suppl.Table 1). In addition, we collected plasma samples from 18 (21

6 episodes) lung transplant recipients (LTRs) with other respiratory viral infections (other

7 RVIs: influenza and respiratory syncytial virus), 21 stable transplant patients with no

8 history of infection and rejection and from 19 healthy controls (HCs) for comparison 9 purposes.

10 cfDNA maps COVID-19 associated tissue injury in SOTRs.

11 Given the ongoing nature of the COVID-19 pandemics and the high risk of severe

12 complications in immunocompromised hosts, a comprehensive tissue-damage mapping

13 in transplant patients infected with COVID-19 is a pressing need to illustrate the extent of

14 tissue injury that correlates with disease severity and outcomes. To do that, we obtained

15 admission plasma samples from 44 SOT COVID-19 patients, as well as plasma from stable

16 SOTRs $(n=21)$ and healthy controls (HCs) $(n=19)$. We first isolated and quantified total

17 plasma cell-free mitochondrial (mtcfDNA) and nuclear DNA (ncfDNA), measures of global

18 cellular death or tissue injury, via digital droplet PCR using mitochondrial and nuclear

19 target primer sets, respectively. cfDNA showed a nucleosomal distribution with a

20 prominent peak length around $\sim 167$ bp (Supp.Fig.1), indicating good quality of cfDNA.

21 We next performed whole-genome bisulfite sequencing and used DNA methylation-based

22 deconvolution algorithm to identify and determine the relative contribution of each tissue 
1 type to the plasma cfDNA ${ }^{19}$. We then computed the absolute copies of tissue-specific

2 cfDNA per $\mathrm{mL}$ plasma $(\mathrm{cp} / \mathrm{mL})$ by multiplying the relative estimated proportions of tissue-

3 specific cfDNA with ncfDNA ( $\mathrm{cp} / \mathrm{mL}$ ) (see Methods) and generated heatmap on log-

4 transformed cfDNA data. The heatmap revealed divergent tissue injury patterns in both

5 SOT and Non-SOT COVID-19 patients (Fig.1 b).

6 The results of our analyses revealed markedly elevated mtcfDNA (median [IQR] =

$710,361,850[3,076,139-32,192,441] \mathrm{cp} / \mathrm{mL}$ ) levels in SOTRs with COVID-19 compared

8 to stable transplant (median $[I Q R]=105,730[51,158-30,4126] \mathrm{cp} / \mathrm{mL}$ ) or healthy controls

9 (median $[I Q R]=30,334[22,995-66,624] \mathrm{cp} / \mathrm{mL}) \quad(p<0.05$ and FDR<0.25; Fig.2a).

10 Similarly, the absolute copy number of ncfDNA was significantly increased in SOT

11 patients with COVID-19 (median $[\mathrm{IQR}]=12,943[6,502-41,546] \mathrm{cp} / \mathrm{mL}$ ) compared to

12 stable transplant (median $[\mathrm{IQR}]=7,485[4,646-23,701] \mathrm{cp} / \mathrm{mL}$ ) or healthy controls

13 (median $[\mathrm{IQR}]=1,218[891-1,870] \mathrm{cp} / \mathrm{mL})(\mathrm{p}<0.05$ and FDR<0.25; Fig.2b). Our

14 deconvolution analysis revealed the majority of circulating cfDNA are derived from

15 hematopoietic origin cells. Specifically, cfDNA derived from monocytes, NK cells, B-cells,

16 neutrophils and erythroblasts were significantly increased in SOTRs with COVID-19

17 compared to stable transplant and healthy controls ( $p<0.05$ and FDR $<0.25$; Fig.2c-g).

18 Likewise, we detect significant injuries from non-hematopoietic tissues $(p<0.01$ and

19 FDR<0.25; Fig.2h-p), including adipocytes, hepatocytes, lung, pancreas, vascular

20 endothelium, cardiac myocytes, and kidney in the bloodstream of SOT COVID-19 patients

21 than in stable transplant and healthy controls. The levels of neutrophil, bladder and

22 squamous epithelium derived cfDNA were significantly increased in SOT COVID-19 
1 patinets relative to healthy controls, but not relative to stable transplant patients. Notably,

2 total cfDNA and neutrophil-derived cfDNA were significantly higher in stable transplant

3 patients compared to healthy controls ( $p<0.05$ and FDR $<0.25$; Fig. $2 b$ and $f$ ), suggesting

4 ongoing tissue injury imposed by the transplant state. This suggests that COVID-19

5 caused systemic injury to multiple cell and tissue types in SOTR with COVID-19.

6 Additionally, our data showed no significant difference in cfDNA levels among SOT types

7 (Suppl.Fig.2a-p). Further, tissue-specific cfDNA measures correlate with established

8 markers of tissue or end-organ injury (Suppl.Fig.3) (see supplementary text for details).

9 Comparable cfDNA profiles between SOT and Non-SOT COVID-19 patients.

10 To examine the effect of COVID-19 on immunosuppressed transplant recipients, we

11 compared cfDNA patterns in these patients to Non-SOT patients matched for COVID-19

12 disease severity median [IQR]: 4 (3-5) vs 4 (3-7), $p=0.087)$. During the COVID-19 illness,

13 SOTs were maintained on tacrolimus and mycofenalate was discontinued. We found that

14 SOT patients with COVID-19 had significantly lower absolute copy number of ncfDNA when compared to those Non-SOT matched COVID-19 patients (median [IQR]: 12,943 $[6,502-41,546] \mathrm{cp} / \mathrm{mL}$ vs 20,979 [12,312 - 42,054] cp/mL; $\mathrm{p}=0.035$ and FDR=0.25), but not mtcfDNA copy number (Fig.3a-b). We then analyzed the level of tissue-specific cfDNA to measure tissue injury patterns (Fig.3c-p). The levels of B cell and cardiac myocyte-

19 derived cfDNA were significantly lower in the SOTRs with COVID-19 as compared with the matched Non-SOT COVID-19 patients ( $p<0.05$ and FDR<0.25; Fig.3d and m). We

21 observed similar injury patterns with the other majority of cell and tissue types, including 22 monocytes, NK cells, neutrophil, erythroblast, vascular endothelium, adipocytes, 
1 hepatocytes, lung and kidney derived cfDNA, when comparing between SOT patients with

2 COVID-19 and Non-SOT COVID-19 comparator groups, although the latter tended to

3 have higher levels of cfDNA ( $p>0.05$ and FDR $>0.25$ ). This suggests a comparable tissue

4 injury pattern between SOT and Non-SOT COVID-19 patients.

\section{Distinct cytokine response in SOTRs with COVID-19.}

6 An excessive and dysregulated cytokine response is associated with severe COVID-19

7 disease ${ }^{31,37}$. To determine whether the immunosuppressed SOTRs with COVID-19 display

8 dysregulated circulatory cytokines signatures, admission plasma levels of 36 cytokines

9 and chemokines were measured (see Methods) in SOTRs with COVID-19 ( $n=44)$ and

10 compared with matched Non-SOT COVID-19 patients $(n=38)$ and healthy controls $(n=30)$.

11 We found altered cytokine levels in both SOT and Non-SOT patients with COVID-19

12 compared to healthy controls $(p<0.05$ and FDR<0.25; Fig.4). Specifically, high plasma

13 levels of IFN- $\lambda 1$, IFN- $\gamma$, IL-1RA, IL-6, MCP-2, and lower levels of Eotaxin-3, IL-12p70, IL-

14 13, MCP-4, MDC, TARC, were observed both in SOT and Non-SOT COVID-19 patients

15 as compared to HCs. The levels of IP-10, IL-15, IL-18, and TNF- $\alpha$ were exclusively

16 increased in SOTRs with COVID-19. Conversely, significantly higher levels of GM-CSF,

17 IFN- $\beta$, IL-10, and IL-2 and low levels of Eotaxin, IL-4, IL-5, MCP-1, and MIP-1 $\beta$, were distinctive to Non-SOT COVID-19 patients relative to HCs, but not in SOTRs. The levels

19 of IFN- $\beta(p=0.059)$, GMCSF $(p=0.099)$ and IL-10 $(p=0.077)$ were higher in SOTRs, whereas in Non-SOT patients IL-1 $\beta(p=0.053), \mathrm{IL}-15(p=0.078)$ and TNF- $\alpha(p=0.053)$ were

21 higher; FDR<0.20. We next compared the cytokine signatures in SOTRs with COVID-19

22 and matched Non-SOT COVID-19 patients. We found higher levels of Eotaxin, GM-CSF, 
1 IFN- $\lambda 1$, IL-13, IL-16, IL-4, IP-10, MCP-1, MCP-2, MCP-4, MDC, MIP-1 $\beta$, TARC, and TNF- $\beta$

2 in SOT COVID-19 patients. These results demonstrate SARS-CoV-2 infection induces

3 distinct cytokine response in SOTRs with COVID-19 compared to Non-SOT COVID-19

4 patients.

5 Integrated cfDNA and cytokine analysis stratify SOT COVID-19 patients based on

6 disease severity.

7 We have previously shown cfDNA derived from both hematopoietic and non-

8 hematopoietic tissues were markedly elevated in in Non-SOT patients with poor COVID-

919 outcomes $^{27}$. Tissue-specific cfDNA measures showed superior performance to identify

10 patients who eventually develop poor COVID-19 outcomes than previously described

11 conventional inflammatory and organ injury biomarkers ${ }^{27}$. Given the heterogeneous

12 nature of the COVID-19 clinical course, we stratified SOT patients by disease severity to

13 test whether admission cfDNA profile correlated with subsequent clinical trajectory in

14 SOTRs. Based on the maximum WHO scale during COVID-19 hospitalization, subjects

15 were categorized as WHO scale 3-4 assigned for mild/moderate COVID-19 ( $n=32$,

$1672.7 \%)$ not requiring ICU, or as WHO scale 5-7 assigned for severe COVID-19 ( $n=12$,

$1727.3 \%)$ requiring ICU care ${ }^{38}$. The data showed that the levels of ncfDNA at admission

18 were significantly increased in SOT patients who eventually developed severe disease

19 compared to those patients with mild/moderate COVID-19 (median [IQR] $=47,708[29,099$

$20-97,667] \mathrm{cp} / \mathrm{mL}$ vs. $8,828[5,815-14,909] \mathrm{cp} / \mathrm{mL}, \mathrm{p}<0.001$ and $F D R<0.05)$, but not

21 mtcfDNA (Fig.5a-b). Deconvolution analyses revealed that SOTRs with severe COVID-19

22 disease displayed distinct tissue injury profiles from those with mild/moderate COVID-19. 
1 They showed significantly increased cfDNA levels derived from monocytes, neutrophils,

2 erythroblast, lung, and pancreas compared to those with mild/moderate disease after

3 adjusting for age, sex, and BMI ( $p<0.05$ and FDR<0.25; Fig.5c, f, g, j and k). Interestingly,

4 the receiver operator characteristic (ROC) curve analysis for the cfDNA features,

5 including neutrophils (AUC $=0.909,95 \%$ confidence interval $[\mathrm{Cl}]=0.816-1.00)$, ncfDNA

$6 \quad(\mathrm{AUC}=0.872,95 \% \mathrm{Cl}=0.756-0.989)$, monocytes $(\mathrm{AUC}=0.813,95 \% \mathrm{Cl}=0.664-0.961)$,

7 erythroblasts $(\mathrm{AUC}=0.810,95 \% \mathrm{Cl}=0.669-0.951)$ and pancreas $(\mathrm{AUC}=0.706,95 \% \mathrm{Cl}$

$8=0.525-0.887)$ showed high to modest performance to identify SOT patients who

9 subsequently develop severe disease/died (Fig.5n, p<0.05).

Next, we investigated whether admission plasma cytokine levels are associated with disease severity ( $p<0.05$ and FDR<0.25; Fig.6a). We observed significantly increased levels of inflammatory cytokines/chemokines such as GM-CSF, IL-15, IL-6 and IL-8 in both SOT and Non-SOT COVID-19 patients who subsquently developed severe disease compared to patients with mild/moderate disease. The levels of Eotaxin, Eotaxin-3 and IL10 were exclusively elevated in severe SOT COVID-19 whereas IL-16, IL-1RA, IL-1a, MIP$1 \alpha$ and TNF- $\alpha$, were solely increased in Non-SOT COVID-19 patients with severe disease, as compared to their respective mild/moderate group. Although they did not reach statistical significance, IL-1 $\alpha(p=0.095$ and FDR=0.29), IL-1 $(p=0.071$ and FDR=0.26) and MCP-1 $(p=0.051$ and FDR=0.23) and were higher in SOTRs with severe disease while IFN- $\alpha 2 a(p=0.082$ and FDR=0.27) and IFN- $\beta(0.064$ and FDR=0.26) were lower. Similarly, Eotaxin $(p=0.080), I L-10(p=0.095), I L-18(p=0.095)$ and IL-2Ra $(p=0.071)$ were higher and IFN- $\beta$ ( $p=0.094)$ was lower in Non-SOT COVID-19 patients (FDR $<0.26$ for all). The ROC 
1 curve analysis of each cytokine profile showed modest performance relative to cytokine

2 features to identify SOT patients who develop severe disease, with IL-15, IL-8, IL-10, GM-

3 CSF and Eotaxin-3 yielding significant AUC performance of $0.836[95 \% \mathrm{Cl}=0.712-$

$40.953], 0.789[95 \% \mathrm{Cl}=0.650-0.923], 0.766[95 \% \mathrm{Cl}=0.622-0.909], 0.770[95 \% \mathrm{Cl}=$

$5 \quad 0.756-0.989]$, and $0.707[95 \% \mathrm{Cl}=0.523-0.891]$, respectively; Fig.6b. The random

6 forest analysis also identified the most discriminative cfDNA (ncfDNA, neutrophil,

7 monocyte, adipocyte, and erythroblast) and cytokine (IL-15, IL-16, IL-23p40, TNF- $\alpha$, and

8 IL-6) features at admission for predicting disease severity in SOT patients with high

9 performance (AUC $=0.875(95 \% \mathrm{Cl}$ : 0.765-0.985) (Suppl.Fig.4a-d).These results

10 demonstrate that integrated cfDNA and cytokine profile may serve as a sensitive approach

11 to identify SOTRs who eventually progressed to severe disease. Furthermore, longitudinal

12 cfDNA and pro-inflammatory cytokine levels remained elevated in SOT COVID-19 patient

13 who eventually died, whereas gradually decreased with time in SOT patients with

$14 \mathrm{mild} /$ moderate disease who eventually recovered (Suppl.Fig.5) (see supplementary

15 materials for details).

16 Correlation of cfDNA profiles and cytokine signatures.

17 To understand the relationship between the host inflammatory responses and tissue injury 18 pattern, we calculated the Spearman's correlation between all biomarkers pairs (i.e., 19 cfDNA profiles and cytokines) across patients within the SOT COVID-19 and Non-SOT 20 COVID-19 groups. To find biomarkers that are highly correlated with each other, we 21 applied unsupervised clustering to group the biomarkers into ten clusters. We found the 22 size and components of the clusters are different between SOTRs and Non-SOT COVID- 
119 patients (Fig.7a and b), as well as disease severity subgroups (see supplementary 2 materials for details, Suppl.Fig.6 and 7)). While IL-15 and IL-16 are clustered with 3 neutrophil-derived cfDNA in SOTRs with COVID-19, inflammatory cytokines such as IL-

4 15, IL-6, IL-16, and MIP-1a are clustered with total (ncfDNA) and tissue-specific cfDNA 5 from adipocytes, monocytes, erythroblasts, and neutrophils in Non-SOT COVID-19 6 patients. The Spearman's correlation among the biomarkers revealed that global and 7 tissue-specific cfDNA profiles were highly correlated with several cytokine signatures. 8 Specifically, ncfDNA was correlated with cytokines/chemokines such as IL-15, IL-8, 9 Eotaxin-3, IL-1 $\beta$, IL-10, IL-16, VEGF, IL-6, IL-7, GM-CSF and IL-1 $\alpha$ in SOTRs with COVID1019 (Fig 7A, Suppl.Table 2). In Non-SOT patients (Fig7b, Suppl.Table 3), while TNF-a, 11 IL-18 and IL-2Ra were significantly correlated with ncfDNA, MCP-2, GM-CSF, IL-6, IL-10 12 and IL-8 were marginally correlated. Given the innate immune cells are linked to COVID1319 immunopathogenesis ${ }^{39}$, our analysis revealed monocyte-derived cfDNA showed the 14 strongest correlation with cytokines associated with cytokine release syndrome (CRS $)^{40}$ 15 such as IL-15, IL-6, IL-8, IP-10, IL-10, GM-CSF, Eotaxin-3, IL-1ß, IL-1RA, IL-18, MCP-2, 16 MCP-1, VEGF, IL-1 $\alpha$, IL-7, TNF- $\alpha$, IL-12p70, and IL-17A in SOTRs with COVID-19 (Fig. 17 7c). Similarly, neutrophil-derived cfDNA was strongly correlated with IL-16, IL-15, IL-8, 18 MCP-1, Eotaxin-3, IL-10, MIP-1 $\beta$, GM-CSF, and IL-1 $\beta$ (Fig.7d). In Non-SOT COVID-19 19 patients (Suppl.table 3), we observed 6 (IL-13, IL-10, IFN-ү, IL-12p70, MCP-2, IL-6) and 20 9 (IL-16, IL-13, TNF-a, IL-6, IL-18, IL-15, MIP-1a, IL-2Ra, IL-8) cytokine correlations with 21 monocyte and neutrophil derived cfDNA, respectively. Additionally, we observed 3 (IL-7, 22 IL-6 and IL-17A) and 1 (IL-17A) correlations between NK cell-derived cfDNA and 
1 cytokines; 1 (Eotaxin-3) and 2 (MIP-1 $\alpha$ and IL-17A) correlations between B cell-derived

2 cfDNA and cytokines; and 5 (IL-15, TNF- $\beta$, IL-7, IL-8, and VEGF) and 1 (IL-13) correlations

3 between erythroblast-derived cfDNA and cytokines levels in SOT and Non-SOT COVID-

419 patients, respectively. Among non-hematopoietic tissues, we observed 7 (IL-4, TNF-a,

5 IL-6, IL-10, IP-10, IL-18, MIP-1 $\alpha$ ) and 2 (IL-16 and IL-6 ) correlations between adipocyte-

6 specific cfDNA and cytokines, 1 (IL-7) and 0 correlations between hepatocyte-specific

7 cfDNA and cytokine, 2 (IL-7 and TARC) and 1 (IL-17A) correlations between and vascular

8 endothelial cell-derived cfDNA and cytokines, 6 (Eotaxin-3, IL-15, IL-8, IL-7, VEGF, IL-16)

9 and 3 (IFN- $\alpha 2 a$, MCP-4, IL-1 $\beta$ ) correlation between pancreas-specific cfDNA and

10 cytokines, 4 (IL-18, IL-15, IL-1 $\beta$, IP-10) and 4 (IL-6, GM-CSF, MCP-2, IL-1 $\beta$ ) correlation

11 between squamous epithelial cell-derived cfDNA, and 0 and 5 (IL-15, IFN- $\beta$, IL-16, IP-10,

12 IL-10) correlation between kidney and cytokines levels in SOTRs and Non-SOT COVID-

1319 patients, respectively (Suppl.Table 2 and 3). The other cell or tissue types showed no

14 significant correlation with cytokine levels in both groups. These observations provide

15 evidence of distinct interaction of host immune response and cfDNA profiles between

16 SOTRs with COVID-19 and Non-SOT patients.

17 To further explore the potential association between circulating cytokine levels and the extent of global and tissue-specific injury profiles, we applied a linear regression analysis

19 to test the association between each cytokine and each cfDNA feature (see methods)

20 (Fig.8a and b, suppl.Fig.8, suppl.Table 4 and 5). The t-statistics from the coefficient of

21 the linear regression are shown in Figure $8 a$ and b. Significant associations (FDR $<0.25$ )

22 are marked with stars. The results showed that cytokines/chemokines such as IL-8, IL-15, 
1 IL-6, IL-7, IL-1 $\beta$, Eotaxin-3, IL-10, IL-4, MIP-1 $\beta$, IL-12p70 and VEGF were associated with

2 global tissue injury profile, i.e., ncfDNA, in SOTRs with COVID-19, whereas TNF- $\beta$, TNF-

3 a, IL-18, IL-6, IL-13, IL-2Ra, MCP-2, and IL-10 were associated with global tissue injury in

4 Non-SOT patients. More importantly, monocyte-derived cfDNA was not clustered with

5 other cfDNA parameters and associated with most of the proinflammatory cytokines and

6 chemokines in SOTRs with COVID-19 (Fig8a, Fig.8c). The strongest association was

7 found with MCP-1 (t-statistics= 6.2, $p=2.29 \mathrm{E}-07$ ), MIP-1 $\beta$ (t-statistics= 6.1, p=2.46E-07),

8 IP-10 (t-statistics= 5.9, p=5.97E-07), IL-18 (t-statistics $=5.4, p=2.96 E-06), \quad M D C$ (t-

9 statistics $=5.0, p=1.23 \mathrm{E}-05), \mathrm{IL}-8$ (t-statistics $=3.9, \mathrm{p}=0.0003)$, TARC (t-statistics $=3.9$,

$10 p=0.0004)$, IL-1RA (t-statistics $=3.5, p=0.001), I L-6$ (t-statistics $=3.4, p=0.0015), M C P-4$ (t-

11 statistics $=3.3, p=0.0019), I L-15$ (t-statistics $=3.1, p=0.004), I L-2 R a$ (t-statistics $=2.8$,

$12 \mathrm{p}=0.007$ ), IL-10 (t-statistics $=2.8, \mathrm{p}=0.008), \mathrm{MCP}-2$ (t-statistics $=2.8, \mathrm{p}=0.008$ ). Levels of

13 Eotaxin-3, MIP-1a, IL-12p70, TNF- $\alpha$, IL-7, GM-CSF, and IL-23p40 were also significantly

14 associated with monocyte-derived cfDNA in SOTRs with COVID-19 ( $p<0.05$ and

15 FDR $<0.25)$. Conversely, monocyte-derived cfDNA was co-clustered with cfDNA derived

16 from neutrophils, erythroblast, adipocytes, and NK cells and negatively associated with

17 IL-13 in Non-SOT patients (Fig.8b). Likewise, neutrophil-derived cfDNA was associated

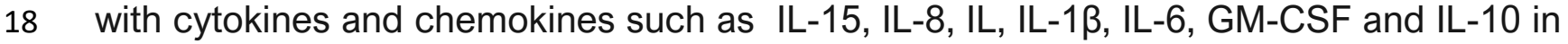

19 SOT patients (Fig.8d), whereas in Non-SOT patients neutrophil-derived cfDNA was

20 negatively associated with IL-13. Additionally, the following association has been

21 observed in SOTRs with COVID-19: adipocyte-derived cfDNA with TNF-a, IL-2, IL-6, IL-4,

22 MIP-1a, and IL-5; vascular endothelium-derived specific cfDNA with IL-7, TARC, MCP-4; 
1 pancreas-derived cfDNA with Eotaxin-3, IL-7, IL-8, IL-15, IL-1 $\beta$, and VEGF; and ddcfDNA

2 with IL-8. On the other hand, Non-SOT COVID-19 patients showed the following

3 associations: NK cells with IL-17A; Adipocyte with IL-12p70 and IL-13; and Kidney with

4 IFN- $\beta$ (positively) and [IL-15, IP-10, TNF- $\alpha$, IL-10, MCP-2, and Eotaxin-3; negatively) has

5 been significantly correlated in non-transpant COVID-19 patients. Collectively, these

6 findings suggested that monocyte-macrophage cell lineages and neutrophils are the

7 major players of COVID-19 immunopathology.

8

9

10

11

12

13

14

15

16

17

18

19

20

21

COVID-19 shows distinct tissue injury profiles compared to other respiratory viruses.

COVID-19, like other RVIs such as influenza A and B, respiratory syncytial virus, parainfluenza, and rhinovirus are associated with significant morbidity and mortality in SOTRs, particularly among LTRs ${ }^{41-43}$. However, the extent of tissue injury of SARS-CoV-2 compared to other RVIs has not been studied previously. We compared the cfDNA profile in LTRs with SARS-CoV-2 $(n=21)$ to LTRs with other RVIs $(n=21)$. The median mtcfDNA level was higher in LTRs with COVID-19 (median [IQR] $=5,580,254[2,435,676$ $13,148,455] \mathrm{cp} / \mathrm{mL}$ ) than for LTRs with other RVls (median $[\mathrm{IQR}]=113,985[75,197-$ 156,411] cp/mL) (Fig.9a, p<0.0001) matched for disease severity using WHO Ordinal Scale. The ncfDNA level was marginally higher in LTRs with COVID-19 $(8,911$ [4,603 $19,881] \mathrm{cp} / \mathrm{mL}$ vs $16,483[6,789-46,123] \mathrm{cp} / \mathrm{mL}$, Fig.9b, p=0.0646) as compared with LTRs with other RVIs. Interestingly, the tissue-specific cfDNA levels were different, with LTRs with COVID-19 showing increased levels of ncfDNA from monocytes, B cells, NK cells (Fig.9c-e), vascular endothelium (Fig.9j), pancreas (Fig.9k), bladder (Fig.9i), and 
1 lung (Fig.9o) in their plasma compared to other RVls; $p<0.05$ for all). There was no

2 significant difference in other cell or tissue types.

Allograft injury is elevated in COVID-19 SOTRs.

4 We demonstrated previously that plasma ddcfDNA fraction (\%) is a sensitive biomarker

5 for allograft injury, and detects allograft rejection and predict long-term outcomes in

6 SOTRs ${ }^{22,23}$. The ddcfDNA released from transplanted organs into the recipient circulation

7 is not specific to allograft rejection; elevations have been observed during viral infections

8 and other pathological conditions ${ }^{44}$. Here we explored whether ddcfDNA could detect

9 allograft injury in SOTRs infected with SARSCOV2. We found that \%dd-cfDNA was

10 significantly higher (median $[\mathrm{IQR}]=1.34 \%[0.41-2.59 \%]$ ) in SOTRs with COVID-19 than

11 stable transplant controls (median $[\mathrm{IQR}]=0.13 \%[0.067-0.25 \%])($ Fig.10a, $p<0.001)$.

$1279.5 \%$ of the COVID-19 transplant patients showed higher ddcfDNA levels than median

13 stable transplant values, including $83 \%$ of lung, $66.7 \%$ of kidney, $66.7 \%$ of the heart, all

14 the liver, and one multiorgan transplant patient. Although it didn't reach statistical

15 significance, the fraction of ddcfDNA tended to be higher in patients who develop severe

16 disease compared to mild cases (median[IQR]: $1.52 \%$ [0.69-2.46] vs $0.97 \%$ [0.19 - 4.5]

17 (Fig.10b). Previous studies showed the ddcfDNA fraction calculation is influenced by the 18 total recipient-derived cfDNA concentration and COVID-19 patients release excessive 19 total cfDNA ${ }^{45}$; this may mask the intensity of allograft injury. The ddcfDNA test used in this 20 study does not incorporate absolute dd-cfDNA levels and consideration of total cfDNA 21 levels in assessing allograft injury may show a more exuberant allograft injury with COVID-

22 19. Although ddcfDNA level didn't show significant difference, the median \%ddcfDNA 
1 tends to higher in LTRs with COVID-19 (median [IQR] $=1.6 \%[0.76-3.79]$ ) as compared

2 with other SOT types with COVID-19 (median [IQR] $=0.71 \%[0.19-2.79]$ or to LTRs with

3 other RVIs (median [IQR] =1.37\% [0.42 - 5.8]; Fig. $10 \mathrm{c}$ and d, $p>0.05$ for both comparisons.

4 An additional finding from this study is ddcfDNA was strongly correlated with plasma

5 cytokines/chemokine such as IL-10 ( $r=0.43)$, IL-8 ( $r=0.43)$, IL-2Ra ( $r=0.39)$, IL-18 ( $r=0.37)$,

6 GM-CSF ( $r=0.36)$ and IL-12p70 ( $r=0.35)$, and marginally correlated with levels of IL-15

$7 \quad(r=0.30, p=0.07), I L-1 \alpha(r=0.29, p=0.08) ;(F i g .10 e, F D R<0.25$ for all). ddcfDNA were also

8 correlated with cfDNA levels derived from NK-cells $(r=0.54)$, monocytes $(r=0.50)$,

9 erythroblast $(r=0.49)$, and marginally correlated with neutrophil $(p=0.069)$ and $B$-cell

10 ( $p=0.07)$; (Fig.10f, FDR<0.25 for all. We observe that COVID-19 triggers allograft injury,

11 even for allografts distant from the primary site of SARS-COV-2 infection. The correlation

12 of allograft injury to cytokine levels suggests that dysregulated cytokine response and

13 immune cells may potentially contribute to allograft injury. 


\section{Discussion}

2 Based on prior experience with other respiratory viral infections, SOTRs are at higher risk

3 of severe complications and deaths ${ }^{41-43}$. Early identification of tissue injury, including

4 allograft injury, as well as inflammatory response, may further illucidate disease

5 pathogenesis, guide risk stratification, inform therapeutic options to improve long-term

6 outcomes of SOTRs. This study performed the first integrated cfDNA and cytokine

7 analysis to elucidate the tissue injury patterns and immune response in SOTRs with

8 COVID-19, including a direct comparison with immunocompetent patients and SOTRs

9 with other respiratory viruses. We leveraged a biomarker of the tissue injury -cell-free

10 DNA - to measure allograft injury as ddcfDNA and injury from different tissue types using

11 cell/tissue-specific DNA methylomic signatures. The tissue-specific cfDNA measures

12 correlated with known markers of tissue injury, indicating that this cfDNA approach

13 reliably measures tissue injury. In accordance with the known systemic clinical

14 manifestations of COVID-1946, we found evidence of tissue injury from diverse cell or

15 tissue types, including hematopoietic cells, vascular endothelium, adipocyte, pancreas,

16 hepatocyte, kidney, heart and lung. cfDNA from these tissue types also showed high

17 performance to identify patients who subsequently developed severe disease/died.

18 Moreover, we analyzed immune responses of SOTRs with COVID-19 using multiplex

19 cytokine profiling in the bloodstream of patients and correlated them with cfDNA profiles.

20 Our analysis of circulating cytokine and chemokine signatures revealed distinct patterns

21 in SOTRs with COVID-19 and the majority of the cytokines were correlated with

22 monocyte-derived cfDNA. Additionally, IFN- $\lambda 1$, a type III interferon, was markedly 
1 elevated in SOTRs with COVID-19 compared to Non-SOT COVID-19 patients. This was

2 an unexpected finding as IFN- $\lambda 1$ and type I interferons are thought to be suppressed in

3 SARS-CoV-2 infections ${ }^{47}$. Moreover, IFN- $\lambda 1$ is actually higher in influenza infection than

4 in SARS-CoV-2 ${ }^{31}$.

5 COVID-19 is caused by a complex interplay between direct SARS-CoV-2 infection and

6 subsequent inflammatory responses ${ }^{48,49}$. However, there are conflicting reports on the

7 impact of immunosuppressive drugs on the severity of COVID-19 in transplant

8 recipients ${ }^{12,50-53}$. Most of these studies are based on traditional inflammatory and organ

9 injury markers, and definitive diagnoses with these conventional biomarkers are

10 challenging due to their low sensitivity and predictive performance ${ }^{54}$. Circulating cfDNA,

11 which carries genetic and epigenetic information from tissues-of-origin, is a sensitive

12 noninvasive biomarker in multiple clinical settings, such as cancer ${ }^{55}$, infectious

13 diseases, ${ }^{27,28}$ and allograft rejection ${ }^{22}$. The cfDNA measures from various tissue types

14 correlated with disease clinical trajectory and outcome and have superior predictive

15 performance over established laboratory markers ${ }^{27}$. This study performed the first

16 integrated cfDNA and cytokine analysis to elucidate the tissue injury patterns and immune

17 response in SOTRs with COVID-19, including a direct comparison with immunocompetent

18 patients. Our data show that total plasma cell-free nuclear and mitochondrial DNA, a

19 measure of global cellular death and tissue injury, were markedly elevated in SOTRs in

20 contrast to stable transplant patients and healthy controls. Interestingly, our result also

21 demonstrated plasma ncfDNA, was significantly lower in SOTRs in contrast to Non-SOT

22 patients. Importantly, our analysis indicated that ncfDNA levels were significantly higher 
1 in SOT COVID-19 patients who eventually developed severe disease or died, suggesting

2 it is a sensitive marker of global tissue injury. However, the mtcfDNA was an imperfect

3 predictor of disease progression/outcome, and our findings disagree with a recent study

4 that reported mtcfDNA as a predictive marker of poor COVID-19 outcomes in Non-SOT 5 settings ${ }^{56}$.

6 In accordance with previous reports ${ }^{31,37}$, our plasma cytokine profiling revealed ongoing

7 alteration of circulating cytokine levels in both SOTRs and Non-SOT COVID-19 patients.

8 We have identified 11 cytokine/chemokine differences in both SOT and Non-SOT COVID-

919 patients as compared to healthy controls, including cytokines with higher (IFN- $\lambda 1$,

10 MCP-2, IL-6, IL-1RA, IFN-y) or lower (Eotaxin-3, MDC, TARC, IL-13, MCP-4, IL-12p70)

11 levels. While the levels of IP-10, IL-15 and IL-18 were exclusively increased in SOTRs with

12 COVID-19, Non-SOT patients with COVID-19 showed solely higher levels of IFN- $\beta$, IL-2,

13 IL-10, GM-CSF and low levels of Eotaxin, MCP-1, MIP-1 $\beta$, IL-4, IL-5. Additionally, high IFN-

$14 \lambda 1$, GM-CSF, MCP-2, TNF- $\beta$, IL-16 and low eotaxin-3 were seen in SOTRs with COVID-19

15 as compared to Non-SOT patients. The levels of inflammatory cytokines/chemokines such

16 as IL-8, IL-15, IL-10, GM-CSF, IL-6, Eotaxin and Eotaxin-3 were increased in the

17 bloodstream of SOT COVID-19 patients who eventually developed severe disease/died

18 but showed a modest discriminatory performance to distinguish mild/moderate to severe

19 disease. Although an exacerbated inflammatory response is a leading cause of severe

20 COVID-19 immunopathology; the driving factors that lead to excessive inflammation in

21 COVID-19 patients remain poorly defined. The correlation analysis in our study revealed

22 a distinct correlation between cfDNA and cytokine correlations in SOTRs with COVID-19. 
1 Prior studies also reported a bidirectional relationship between cfDNA level and

2 inflammation because cfDNA can trigger a deleterious proinflammatory response. In turn,

3 the inflammation causes excessive cfDNA release, enhancing tissue injury ${ }^{35}$. We have also

4 shown the excessive cfDNA released in COVID-19 patients caused the overproduction of

5 mitochondrial reactive oxygen species, increasing tissue injury ${ }^{27}$. Moreover, elevated

6 plasma cfDNA levels induce coagulation activation and impair fibrinolysis ${ }^{57,58}$, thereby

7 contributing to microvascular thrombosis. Remarkably, IL-13, the prototypical Th2

8 cytokine, was associated with protection against multiple-tissue injury in Non-SOT

9 patients. Nonetheless, these findings suggest that simultaneous immunostimulation and

10 immunosuppression occur in SOT patients that may have a protective or pathologic

11 effect.

12 Although the lung is the primary target organ for SARS-CoV-2, multiple cells, tissue, or

13 organ types can be involved due to broad SARS-CoV-2 cellular tropism ${ }^{59}$ and subsequent

14 inflammatory responses ${ }^{48}$. Our deconvolution results indicated that hematopoietic cells,

15 including granulocytes, erythrocyte progenitors, monocytes, NK cells and B cells, are the

16 predominant source of circulating cfDNA levels in SOTRs with COVID-19. Elevated cfDNA

17 derived from these cell types may be associated with high cellular death/injury from both

18 direct infection with SARS-CoV-2 and indirect effects of systemic inflammation and

19 turnover ${ }^{60,61}$. Neutrophils and monocytes are essential components of innate immunity

20 and appear to play a dominant role in COVID-19 pathogenesis ${ }^{39}$. Our observation also

21 showed neutrophils to be the leading contributor to an elevated cfDNA level in SOTRs

22 with COVID-19. Additionally, neutrophil-derived cfDNA appeared to be an excellent 
1 predictor of progression to severe disease/death. Neutrophils expel cfDNA into the

2 extracellular environment during neutrophil extracellular traps (NETs) formation. The

3 excessive NET formation has been noted in COVID-19 patients and is linked to disease

4 severity ${ }^{62-64}$. Our result also indicated a strong positive correlation between neutrophil-

5 derived cfDNA and D-dimer (a degradation product of fibrin), implicating their role in

6 immunothrombosis. Eotaxin-3, a potent chemoattractant of eosinophils, was markedly

7 decreased in COVID-19 patients, with more elevation in SOTRs with COVID-19. In line

8 with this, admission blood sample analysis revealed eosinopenia in COVID-19 patients

9 who eventually progressed to severe disease/died. Monocytes, a precursor of

10 macrophages, play a causal role in severe COVID-19 immunopathology by driving

11 cytokine storm ${ }^{39}$. We find high monocyte-derived cfDNA in SOTRs with COVID-19 that

12 correlate with subsequent disease severity. Interestingly, we find strong correlations

13 between monocyte-derived cfDNA and proinflammatory cytokines such as TNF- $\alpha$, MCP-

141 , IL-18, and IL-6 in SOTRs as compared to Non-SOT patients. Moreover, our hierarchical

15 linear regression analysis identified monocytes were strongly associated with the majority

16 of inflammatory cytokines in SOT COVID-19 patients. Particularly, our multivariate linear

17 regression analysis showed cytokines/chemokines such as IL-6, IL-8, MIP-1a, GM-CSF,

18 MCP-4, IL-18, TNF- $\alpha$, IL-15, IP-10, MDC, IL-1RA, MCP-1, and MIP-1 $\beta$ as effectors of

19 monocyte injury in SOTRs. Most strikingly, the correlation and linear regression analysis

20 between monocyte-derived cfDNA and cytokines were stronger in SOTRs with severe

21 disease. These findings highlight monocytes as the major contributor to severe

22 immunopathology in transplant patients COVID-19. The current immunosuppressive 
1 regimens (e.g., mycophenolate mofetil and calcineurin inhibitors) are not thought to target

2 the monocyte cell lineages, and the effects on cytokine production are limited ${ }^{65}$.

3 COVID-19 patients also exhibited erythropoiesis aberrations, with increasing erythroid

4 progenitors in the bloodstream of patients with severe disease ${ }^{66}$. Our data corroborated

5 a role for altered erythropoiesis in COVID-19 with elevated circulating cfDNA originating

6 from erythroid progenitors, especially in severe disease. Another feature of COVID-19 is

7 lymphopenia in the peripheral blood ( $T$, B, and NK cells), indicating impairment of

8 adaptive immunity ${ }^{67}$. Absolute lymphocyte count was also low in SOT COVID-19 patients

9 and our methylome analysis corroborated these findings with low/undetectable cfDNA

10 levels from $\mathrm{CD}^{+}{ }^{+} \mathrm{T}$ cells and $\mathrm{CD} 8^{+} \mathrm{T}$ cells. This might be due to the nature of SARS-CoV-

112 infection or the immunosuppressive treatments. A recent study also showed extensive

12 NK cell activation, tissue trafficking, and turnover in COVID-19 patients correlated with

13 subsequent disease progression ${ }^{60}$. Consistent with this study, we found high cfDNA

14 derived from NK cells at admission to be associated with subsequent disease severity.

15 Our work and that of others demonstrate significant injury from non-hematopoietic tissue

16 types in Non-SOT COVID-19 patients ${ }^{27,28}$. Similarly, we found considerable injury from

17 vascular endothelium, adipocytes, liver, pancreas, kidney, heart, and lung in SOTRs with

18 COVID-19 compared to healthy controls and to stable transplant patients. These results

19 suggest the involvement of multiple cell, tissue or organ types in COVID-19 pathogenesis

20 and the results are consistent with clinical presentations of patients with COVID-1929.

21 While we could not find statistically significant differences for the majority of tissue- 
1 specific cfDNA levels in transplant versus Non-SOT patients with COVID-19, B cells and

2 cardiac myocytes-derived cfDNA levels were high in Non-SOT COVID-19 patients.

3 The dd-cfDNA released from the transplanted organ is not specific to rejection, and

4 elevations are also observed during viral infections without concomitant allograft 5 rejection ${ }^{44}$. Viral infections affect allograft function by both direct tissue damage and 6 immunologically induced injury. In this study, we found ddcfDNA was significantly 7 elevated in SOTRs with COVID-19 and strikingly correlated with cytokines/chemokines 8 (IL-10, IL-8, IL-2Ra, IL-18, GM-CSF and IL-12p70) associated with CRS. This suggests the 9 allograft undergoes significant injury following SARS-CoV-2 infection and the subsequent 10 inflammatory response and highlights therapeutic targets to prevent allograft injury. 11 Additionally, the increased circulating cfDNA levels may act as DAMP to activate 12 proinflammatory response through a dsDNA pattern recognition receptor and cause 13 allograft dysfunction ${ }^{68,69}$. Aside from the lung, injury has been detected in transplanted 14 organs distant from the primary site of the infection, such as kidney, liver, and heart. More 15 recently, two case reports showed an increase ddcfDNA in heart and kidney transplant 16 patients with COVID-1970,71. However, total and tissue-specific cfDNA profiles were not 17 different among SOTRs. Similarly, a multicenter clinical study also reported type of 18 transplant was not associated with COVID-19 severity ${ }^{5}$. This supports that COVID-19 is a 19 systemic disease.

20 Another interesting observation from this study was extensive tissue injury in LTRs with 21 COVID-19 as compared with other RVIs. Indeed, histologic analysis of pulmonary vessels 
1 in patients with COVID-19 also showed widespread vascular endothelialitis, thrombosis,

2 and angiogenesis, which distinguishes COVID-19 from severe influenza or respiratory

3 syncytial virus infection ${ }^{46}$. Consistently, we found significantly increased cfDNA levels

4 from the vascular endothelium and immune cells. Although no significant difference was

5 found in allograft injury, measured as \%ddcfDNA between LTRs infected with COVID-19

6 and those with influenza and/or RSV, the cfDNA methylation analysis revealed high levels

7 of lung-derived cfDNA in LTRs with COVID-19. A plausible explanation for this difference

8 is that the \%ddcfDNA calculation is influenced by the total recipient-derived cfDNA

9 concentration ${ }^{45}$, and SOTR COVID-19 patients release excessive total cfDNA. Thus, it may

10 mask the intensity of allograft injury in LTRs with COVID-19 infection. The ddcfDNA test

11 does not incorporate absolute dd-cfDNA levels ${ }^{72}$, and consideration of total recipient-

12 derived cfDNA levels in assessing allograft injury may resolve this discrepancy.

13 There are some limitations that must be noted for this study. First, transplant patients take 14 a combination of immunosuppressive drugs and the net potential effect of each drug type

15 on cfDNA/cytokine level could vary, and these may contribute to intra- and interindividual

16 variability. Second, human tissues are complex, containing a mixture of different cell

17 types, and the deconvolution methods are restricted to predominant cells in each tissue

18 type included in the reference pane ${ }^{19}$. Thus, the result does not account for all cell types

19 and may not be representative of the tissue profile or may have missed clinically relevant

20 information from other cell types of the tissue. In addition, the cause of cellular injury or

21 destruction that results in cfDNA release from diverse tissue types of the body is not clear.

22 Third, the sample size is small due to relatively few cases of transplant patients infected 
1 with SARS-CoV-2, and the under-representation of heart transplant recipients in our

2 cohort. Fourth, SARS-COV-2 variants were not specified in these patients. The tissue

3 injury profiles described here may be different with different SARS-COV-2 variants.

4 Despite these limitations, integrated cfDNA and cytokine analysis captured clinically

5 relevant information in both transplant and Non-SOT populations with COVID-19.

6 In summary, we show that cfDNA is a comprehensive molecular biomarker to map injury

7 from diverse tissue types early in the course of COVID-19 and predicts subsequent clinical

8 trajectory. We have identified distinct cytokine features in transplant versus Non-SOT

9 COVID-19 patients. Additionally, we found significantly elevated allograft injury in SOTRs

10 with COVID-19. In SOTRs, monocyte-derived cfDNA and ddcfDNA were strongly

11 associated with proinflammatory cytokines involved in CRS. Measuring plasma cfDNA

12 early in the course of COVID-19 may help high-risk patients and guide early initiation of

13 appropriate therapies. Further research is needed to further explore the biological

14 interaction between cfDNA and cytokines. These studies may also investigate the clinical

15 utility of early cfDNA measures to guide treatment plan. 


\section{Materials and Methods}

\subsection{Study Subjects and Setting}

A prospective cohort study was conducted at Johns Hopkins Hospital (Clinical Trials.gov identifier $=$ NCT04496466) and University of Maryland Medical Center from April 21, 2020 to September 08, 2021 and include consecutive hospitalized solid organ transplant recipients $(n=44)$ with a confirmed diagnosis of COVID-19 by positive RT-PCR assay for SARS-CoV-2 RNA on a nasopharyngeal swab. All study subjects provided informed consent for blood sample collection at admission and for collection of clinical data, including demographic data, comorbidities, laboratory test results, medications, and other clinical parameters. A subset of patients $(n=6)$ underwent serial blood sample collection during their hospital stay. The specimens utilized for this publication were part of the Johns Hopkins Biospecimen Repository, which is based on the contribution of many patients, research teams, and clinicians. The maximum World Health Organization (WHO) disease severity score reached at any time during the COVID-19 hospitalization was used to categorize patients as mild/moderate (WHO scale 3,4$)$ and severe disease (WHO scale $5-7)^{38}$. The study also collected blood plasma samples from WHO-scale-matched NonSOT COVID-19 patients ( $n=40)$, lung transplant recipients (LTRs) infected with other respiratory virus infections (other RVIs) ( $n=18,21$ episodes), stable transplant recipients without acute rejection or infection $(n=21)$ and healthy controls $(n=30)$ to serve as a comparator group. In our cohort, there were no SOTRs with COVID-19 on extracorporeal membrane oxygenation (ECMO) and we excluded the Non-SOT COVID-19 patients receiving ECMO support at any time course of the disease from the analysis. Patients 
1 without admission plasma samples were also excluded from the analysis. The study was

2 approved by the Institutional Review Boards of the Johns Hopkins University School of

3 Medicine and University of Maryland Medical Center.

4 4.2. Plasma sample processing and cfDNA isolation

5 Plasma samples were isolated from whole blood collected in Cell-Free DNA BCT $\circledast$

6 (Streck, La Vista, NE) or EDTA (BD) tubes by centrifugation at $1600 \mathrm{~g}$ for 10 min at $4^{\circ} \mathrm{C}$,

7 aliquoted and stored immediately at $-80^{\circ} \mathrm{C}$. The aliquoted plasma was thawed at room

8 temperature and centrifuged at $16000 \mathrm{~g}$ for $5 \min$ at $4{ }^{\circ} \mathrm{C}$ to remove residual debris. cfDNA

9 was extracted from $1 \mathrm{~mL}$ of plasma by QIAsymphony circulating DNA kit (QIAGEN). The

10 plasma samples were spiked with $0.142 \mathrm{ng} / \mathrm{mL}$ unmethylated lambda phage DNA

11 (Promega), which was fragmented to $160 \mathrm{bp}$, to measure the efficiencies of cfDNA

12 extraction and bisulfite conversion for genome-wide methylation sequencing. The isolated

13 cfDNA were eluted into $60 \mu \mathrm{L}$ elution buffer and frozen at $-20^{\circ} \mathrm{C}$ until ready for further

14 use.

15 4.3. cfDNA quantification

16 4.3.1. Real-time quantitative PCR (qPCR)

17 The cfDNA was quantified by qPCR for human Alu repeats (Alu115 and Alu247) to check

18 the integrity (ALU247/ALU115). Concurrent qPCR of lamda DNA was used to measure

19 extraction efficiency. Briefly, a $10 \mu \mathrm{l} \mathrm{PCR}$ mixture containing $2 \mu \mathrm{L}$ cfDNA template $(1: 10$

20 diluted), $5 \mu \mathrm{L}$ SYBR Green SuperMix (Bio-Rad), $2 \mu \mathrm{l}$ nuclease-free water, and $1 \mu \mathrm{L}$ primer

21 pair were prepared in triplicate for each amplicon. The PCR reaction mixture was run on

22 QuantStudio 3 (Applied Biosystems) as follows: initial denaturation at $95^{\circ} \mathrm{C}$ for $5 \mathrm{~min}$, 
1 followed by 35 cycles of $95^{\circ} \mathrm{C}$ for $15 \mathrm{~s}$ and annealing at $60^{\circ} \mathrm{C}$ for 1 min using

2 QuantStudio ${ }^{\mathrm{TM}}$ design and analysis software. The primer sequences were as follows:

3 forward, 5'-CCTGAGGTCAGGAGTTCGAG-3' and reverse, 5'-

4 CCCGAGTAGCTGGGATTACA-3' for Alu-115; forward, 5'-GTGGCTCACGCCTGTAATC5 3' and reverse, 5'-CAGGCTGGAGTGCAGTGG-3' for ALU247; and forward, 5'-

6 GACCTCTATGCCAACACAGT-3' and reverse, 5'-AGTACTTGCGCTCAGGAGGA-3' for $\lambda$

7 DNA. The concentrations for each amplicon were calculated using a standard curve

8 generated from 10-fold serially diluted human genomic DNA (Promega), which was

9 fragmented to $160 \mathrm{bp}$. The concentration of short fragments (Alu115) reflects total cfDNA

10 level $(\mathrm{ng} / \mathrm{ml})$, whereas the ratio of shorter fragments (Alu115) to longer fragments

11 (Alu247) was used to estimate DNA integrity. Samples with an integrity score $>0.8$ were

12 eliminated since these are considered to be contaminated with high molecular weight

13 nuclear DNA. The extraction efficiency was calculated as the ratio of input and output of

14 the lambda DNA, which is spiked into plasma samples prior to cfDNA isolation and

15 measured after cfDNA extraction.

\section{4.3.2. Droplet digital PCR (ddPCR)}

17 Absolute copy numbers of ncfDNA and mtcfDNA were quantified on QX 200 Droplet 18 Digital PCR (ddPCR) system, using primers and probes targeting eukaryotic translation 19 initiation factor $2 \mathrm{C} 1$ (EIF2C1) found on the nuclear genome and NADH dehydrogenase 1 20 (ND1) found on the mitochondrial genome, respectively. The probes targeting EIF2C1 21 were labeled with HEX (BioRad, \#10031245) and ND1 probes with FAM (BioRad, $22 \# 10042960)$. The ddPCR was carried out in triplicate of $22 \mu$ reaction volume containing 
$111 \mu \mathrm{L} 2 \mathrm{XddPCR}$ Supermix for Probes (No dUTPs), $4 \mu \mathrm{L}$ of cfDNA (1:10 diluted), and 0.55

$2 \mu \mathrm{L}$ of each $20 \mathrm{X}$ primer/probe sets and $5.9 \mu \mathrm{L}$ of nuclease-free water, followed by 3 partitioning into $\sim 17,000$ droplets. The generated droplets were subjected to PCR

4 amplification at $95^{\circ} \mathrm{C}$ for $10 \mathrm{~min}$, followed by 40 cycles of $15 \mathrm{~s}$ at $95^{\circ} \mathrm{C}$ and $1 \mathrm{~min}$ at 60

$5{ }^{\circ} \mathrm{C}$ with ramp rate set to $2.5^{\circ} \mathrm{C} / \mathrm{s}$ between temperatures. After amplification, the data were

6 acquired using QuantaSoft ${ }^{\mathrm{TM}}$ software on QX200 droplet reader and analyzed using

7 QuantaSoft ${ }^{\mathrm{TM}}$ Analysis Pro software. Absolute copy number of ncfDNA and mtcfDNA were

8 then normalized by plasma volume and extraction efficiency as follows.

$9 \quad$ Copies $/ \mathrm{mL}$ plasma $=($ A copies $/ \mu \mathrm{L}) \times\left[\frac{22 \mu \mathrm{L} \text { of ddPCR rxn }}{\mathrm{B}} \times \mathrm{DF}\right] \times\left[\frac{\text { elution volume }}{\mathrm{C} \text { mL of plasma used } \times \mathrm{EF}}\right]$

Where $\mathbf{A}$ is copies of ncfDNA or mtcfDNA per $[\mu \mathrm{L}]$ as calculated by Quanta soft

11 software, B is the diluted cfDNA volume $[\mu \mathrm{L}]$ used for ddPCR reaction mixture, DF is the

12 dilution factor of the cfDNA sample used for ddPCR reaction mixture, $\mathbf{C}$ the plasma

13 volume $[\mathrm{mL}]$ used for isolation of cfDNA and EF the extraction efficiency as calculated by

14 dividing lambda DNA detected per $[\mu \mathrm{L}]$ over spiked lambda DNA.

15 4.3.3. Donor-derived cell-free DNA

16 Five to $10 \mathrm{ng}$ of isolated cfDNA were subjected to next-generation sequencing (NGS),

17 targeting 405 SNPs, to precisely quantify dd-cfDNA with a range of $0.12 \%-16 \%$, without 18 the need for donor and recipient genotyping ${ }^{73}$.

19 4.4. Library preparation and sequencing

20 Size distribution of the cfDNA was determined using Cell-free DNA ScreenTape assay on

21 Agilent 4150 TapeStation System according to the manufacturer's instructions to assure 
1 the absence of contamination with genomic DNA. An input of 5 - $50 \mathrm{ng}$ of isolated cfDNA,

2 depending on the availability, was used to perform bisulfite conversion using EZ DNA

3 methylation-Gold kit (Zymo Research) as per the manufacturer's recommendation.

4 Libraries were prepared using the Accel-NGS Methyl-Seq DNA Library Kit with Unique

5 Dual Indexing (Swift Biosciences) for whole-genome bisulfite sequencing according to the

6 manufacturer's instructions. The quality of the constructed cfDNA library was visualized

7 using a high-sensitivity D1000 ScreenTape and quantified using the Quant-iT PicoGreen

8 dsDNA Assay kit (Life Technologies). The DNA libraries were then normalized in

9 equimolar concentrations and were subjected for $\sim 200 \mathrm{M}$ reads by $2 \mathrm{X} 100 \mathrm{bp}$, paired-end

10 DNA sequencing on the lllumina NovaSeq 6000.

11 4.5. cfDNA sequence analysis

12 The raw sequence reads were quality checked with FastQC ${ }^{74}$ and trimmed using

13 trim_galore ${ }^{75}$. Adapter sequences and 10 base pairs from both pair-end reads were

14 (paired-end reads $>50 \mathrm{bp}$ were retained). The paired-end sequence reads were

15 subsequently aligned to a C-to-T converted human reference genome (hg38 assembly)

16 using Bismark ${ }^{76}$. Duplicate sequence reads were also removed and post alignment quality

17 control checked using Bismark. The Bismark methylation extractor routine determines

18 cytosine methylation status and extracts all CpGs in each sample. We built a custom

19 bisulfite-sequencing analytic workflow for analyzing and visualizing the cfDNA methylome

20 data using a collection of tools from bsseq $^{77}$. A reference meth_atlas algorithm was used

21 to deconvolute the composition of plasma cfDNA. The reference meth-atlas algorithm is

22 constructed using $\mathrm{CpG}$ methylation dataset of 25 human cell or tissue types. The method 
1 covers in silico simulations of in vitro mixes of known proportion of cfDNA derived from

2 different tissue types. The cfDNA methylation levels of $\mathrm{CpG}$ sites represented as a linear

3 combination of those of 25 cell or tissues types and effectively identify and determine the

4 relative contributions of different cell or tissue types to the plasma cfDNAs with $\sim 4,000$

$5 \mathrm{CpGs}^{19}$. We filtered $\mathrm{CpGs}$ with at least $5 \mathrm{x}$ coverage in individual samples for the

6 deconvolution analysis for each cfDNA sample. The deconvolution algorithm scripts

7 provided at https://github.com/nloyfer/meth_atlas and the methylation analysis scripts

8 at https://github.com/seifudd/cfMethylome. Absolute copies of cell, tissue or organ-

9 specific cfDNA per $\mathrm{mL}$ plasma $(\mathrm{cp} / \mathrm{mL})$ were calculated by multiplying the relative

10 estimated proportions of each cell, tissue or organ type by absolute ncfDNA level $(\mathrm{cp} / \mathrm{mL})$.

11 4.6. Cytokine measurement

12 Cytokines and chemokines (IFN- $\alpha 2 a$, IFN- $\beta$, IL-18, IL-1RA, IFN- $\lambda 1$, IL-2Ra, MCP- 2, GM13 CSF, IL-23p40, IL-15, IL-16, IL-17A, IL-1 $\alpha$, IL-5, IL-7, TNF- $\beta$, VEGF, Eotaxin, Eotaxin-3, IP-

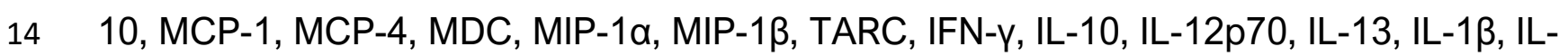
15 2, IL-4, IL-6, IL-8, TNF- $\alpha$ ) were measured in plasma using a custom multiplex kit from 16 Meso Scale Diagnostics (MSD, Rockville, MD) according to the manufacture's protocol.

17 Raw data were acquired using a MESO QuickPlex SQ 120. Each sample was measured 18 in duplicate. If an analyte signal was below background, it was set to 0 . If detectable, but 19 below the manufacturer's lower limit of quantification, it was set to the lower limit of 20 detection. 


\subsection{Statistical analysis}

2 The data were presented as the median (interquartile ranges [IQR]) for continuous

3 variables and as frequency (percentage) for categorical data. Statistical difference

4 between groups was calculated using the Wilcoxon-Mann-Whitney test and Fisher's exact

5 test to compare continuous variables and dichotomized variables, respectively.

6 Univariable and multivariate logistic regression analysis was performed to assess

7 associations between cfDNA levels and patient characteristics, adjusting for age, gender,

8 self-reported race, and BMI. To evaluate the predictive power of cfDNA and cytokine

9 features for the severity of disease, we constructed a random forest model for SOT

10 patients to classify patients with mild/moderate and severe diseases. The predictive

11 performance of cfDNA profile was evaluated using the receiver operating characteristic

12 (ROC) curve. The ROC curve is plotted as sensitivity (true positive rate) and against 1-

13 specificity (false positive rate) and the accuracy was measured by the area under the

14 curve (AUC). An AUC value of 1 represents a perfect predictive power, whereas an AUC

15 of 0.5 indicates no predictive power. The relative importance for each cfDNA feature was

16 assessed using the mean increase in error rate (decrease in accuracy) over all out-of-bag

17 cross-validated predictions.

18 To compare the cytokines between patient groups, a linear regression analysis was

19 performed after adjusting for age, gender, and BMI. P-values were obtained from the

20 model and converted to false discovery rates (FDR) using the Benjamini-Hochberg (BH)

21 procedure ${ }^{78}$. To characterize the connection between the cfDNA levels and cytokines, we

22 calculated Spearman's correlation coefficients among all cfDNA features and cytokines. 
1 To further study the association between cytokines and the origin of tissue damage, we

2 performed a linear regression analysis of the cytokine and cfDNA data. For each cytokine

3 and each cfDNA feature, we fitted a linear regression model using the cytokine as the

4 independent variable and the cfDNA feature as the dependent variable. The t-statistics

5 from the coefficient of linear regression model is used to represent the association

6 between a cytokine and a cfDNA feature. Statistical analyses were carried out in R

7 software version 3.6.3 and GraphPad Prism software version 9. A p-value $\leq 0.05$ and

8 FDR $\leq 0.25$ was considered statistically significant;: FDR $\leq 0.25$ and $p$-value $\leq 0.05$, **: FDR

$9 \leq 0.1$ and $p$-value $\leq 0.05,{ }^{* * *}$ : FDR $\leq 0.05$ and $p$-value $\leq 0.05$, NS: FDR $>0.25$ or p-value $>$ $10 \quad 0.05$. 


\section{Acknowledgements}

2 This work was supported by the Intramural Research Program of the NIH through the

3 Intramural Targeted Anti-COVID-19 grant, Lasker Clinical Research Fellowship Program

4 and Cystic Fibrosis Foundation (grant AGBORE20Q10). COVID-19 specimens utilized for

5 this publication were part of biorepositories established at Johns Hopkins Hospital and

6 University of Maryland Medical Center. We thank the dedicated contributions of many

7 patients, research teams, and clinicians who contributed to creating the biorepository. We

8 also thank Kelly Byrne for editorial support.

9 Competing interests

10 The authors declare no competing interests.

\section{Author contributions}

12 TEA, ALC and SAE conceived and designed the experiment. TEA, HK, and MKJ

13 conducted whole-genome bisulfite sequencing experiment. AHK and AGR acquired

14 cytokine data. FS, KS and MP analyzed whole-genome bisulfite sequencing data. RW

15 conducted next-generation targeted sequencing assay and analyze the data. TEA wrote

16 the manuscript. ALC, RKA and IA recruited patients and provided samples and clinical

17 data. TEA, WZ, TL and JH performed statistical analysis. All authors participated in

18 preparation of the manuscript and gave final approval for publication.

19 Data Availability

20 The datasets generated in this study study are available from the corresponding author 21 upon reasonable request. 


\section{References}

21 Loupy, A. et al. Organ procurement and transplantation during the COVID-19

3 pandemic. Lancet 395, e95-e96, doi:10.1016/s0140-6736(20)31040-0 (2020).

42 Grattan, M. T. et al. Cytomegalovirus infection is associated with cardiac allograft rejection and atherosclerosis. Jama 261, 3561-3566 (1989).

63 Raja, M. A. et al. COVID-19 in solid organ transplant recipients: A systematic review and meta-analysis of current literature. Transplant Rev (Orlando) 35, 100588, doi:10.1016/j.trre.2020.100588 (2021).

94 Weikert, B. C. \& Blumberg, E. A. Viral infection after renal transplantation: Nephrology 3, S76-S86 (2008).

125 Kates, O. S. et al. COVID-19 in solid organ transplant: A multi-center cohort study. Clin Infect Dis, doi:10.1093/cid/ciaa1097 (2020).

146 Garibaldi, B. T. et al. Patient Trajectories Among Persons Hospitalized for COVID19 : A Cohort Study. Ann Intern Med 174, 33-41, doi:10.7326/m20-3905 (2021).

167 Fung, M. \& Babik, J. M. COVID-19 in Immunocompromised Hosts: What We Know So Far. Clin Infect Dis 72, 340-350, doi:10.1093/cid/ciaa863 (2021).

188 Eren Sadioğlu, R. et al. Antibody response to two doses of inactivated SARS-CoV2 vaccine (CoronaVac) in kidney transplant recipients. Transpl Infect Dis, e13740, doi:10.1111/tid.13740 (2021). 
19 Boyarsky, B. J. et al. Antibody Response to 2-Dose SARS-CoV-2 mRNA Vaccine Series in Solid Organ Transplant Recipients. Jama 325, 2204-2206, doi:10.1001/jama.2021.7489 (2021).

10 Marion, O. et al. Safety and Immunogenicity of Anti-SARS-CoV-2 Messenger RNA Vaccines in Recipients of Solid Organ Transplants. Ann Intern Med 174, 13361338, doi:10.7326/m21-1341 (2021).

11 Mlcochova, P. et al. SARS-CoV-2 B.1.617.2 Delta variant replication and immune evasion. Nature, doi:10.1038/s41586-021-03944-y (2021).

12 Colmenero, J. et al. Epidemiological pattern, incidence, and outcomes of COVID19 in liver transplant patients. J Hepatol 74, 148-155, doi:10.1016/j.jhep.2020.07.040 (2021).

13 Boyarsky, B. J. et al. Antibody response to 2-dose SARS-CoV-2 mRNA vaccine series in solid organ transplant recipients. Jama 325, 2204-2206 (2021).

14 Duvvuri, B. \& Lood, C. Cell-Free DNA as a Biomarker in Autoimmune Rheumatic Diseases. Front Immunol 10, 502, doi:10.3389/fimmu.2019.00502 (2019).

15 Oellerich, M. et al. Liquid biopsies: donor-derived cell-free DNA for the detection of kidney allograft injury. Nat Rev Nephrol 17, 591-603, doi:10.1038/s41581-02100428-0 (2021).

16 Ignatiadis, M., Sledge, G. W. \& Jeffrey, S. S. Liquid biopsy enters the clinic implementation issues and future challenges. Nat Rev Clin Oncol 18, 297-312, doi:10.1038/s41571-020-00457-x (2021). 
117 Breveglieri, G., D'Aversa, E., Finotti, A. \& Borgatti, M. Non-invasive Prenatal Testing Using Fetal DNA. Mol Diagn Ther 23, 291-299, doi:10.1007/s40291-019-00385-2 (2019).

418 Polina, I. A., llatovskaya, D. V. \& DeLeon-Pennell, K. Y. Cell free DNA as a diagnostic and prognostic marker for cardiovascular diseases. Clin Chim Acta 503, 145-150, doi:10.1016/j.cca.2020.01.013 (2020).

19 Moss, J. et al. Comprehensive human cell-type methylation atlas reveals origins of circulating cell-free DNA in health and disease. Nature communications 9, 1-12 (2018).

20 De Vlaminck, I. et al. Circulating cell-free DNA enables noninvasive diagnosis of heart transplant rejection. Science translational medicine 6, 241ra277-241ra277 (2014).

21 De Vlaminck, I. et al. Noninvasive monitoring of infection and rejection after lung transplantation. Proceedings of the National Academy of Sciences 112, 1333613341 (2015).

22 Agbor-Enoh, S. et al. Cell-Free DNA to Detect Heart Allograft Acute Rejection. Circulation 143, 1184-1197, doi:10.1161/circulationaha.120.049098 (2021).

23 Agbor-Enoh, S. et al. Donor-derived cell-free DNA predicts allograft failure and mortality after lung transplantation. EBioMedicine 40, 541-553 (2019).

24 Snyder, M. W., Kircher, M., Hill, A. J., Daza, R. M. \& Shendure, J. Cell-free DNA comprises an in vivo nucleosome footprint that informs its tissues-of-origin. Cell 164, 57-68 (2016). 
125 Lehmann-Werman, R. et al. Identification of tissue-specific cell death using methylation patterns of circulating DNA. Proceedings of the National Academy of Sciences 113, E1826-E1834 (2016).

26 Sun, K. et al. Orientation-aware plasma cell-free DNA fragmentation analysis in open chromatin regions informs tissue of origin. Genome Res 29, 418-427, doi:10.1101/gr.242719.118 (2019).

27 Andargie, T. E. et al. Cell-free DNA maps COVID-19 tissue injury and risk of death, and can cause tissue injury. JCl insight (2021).

28 Cheng, A. P. et al. Cell-free DNA tissues of origin by methylation profiling reveals significant cell, tissue, and organ-specific injury related to COVID-19 severity. Med 2, 411-422. e415 (2021).

29 Gupta, A. et al. Extrapulmonary manifestations of COVID-19. Nat Med 26, 10171032, doi:10.1038/s41591-020-0968-3 (2020).

30 Dorward, D. A. et al. Tissue-Specific Immunopathology in Fatal COVID-19. Am J Respir Crit Care Med 203, 192-201, doi:10.1164/rccm.202008-3265OC (2021).

31 Karaba, A. H. et al. Differential Cytokine Signatures of SARS-CoV-2 and Influenza Infection Highlight Key Differences in Pathobiology. Clin Infect Dis, doi:10.1093/cid/ciab376 (2021).

32 Del Valle, D. M. et al. An inflammatory cytokine signature predicts COVID-19 severity and survival. Nat Med 26, 1636-1643, doi:10.1038/s41591-020-1051-9 (2020). 
133 Gould, T. J. et al. Cell-Free DNA Modulates Clot Structure and Impairs Fibrinolysis in Sepsis. Arterioscler Thromb Vasc Biol 35, 2544-2553, doi:10.1161/atvbaha.115.306035 (2015).

34 von Meijenfeldt, F. A. et al. Elevated Plasma Levels of Cell-Free DNA During Liver Transplantation Are Associated With Activation of Coagulation. Liver Transp/ 24, 1716-1725, doi:10.1002/lt.25329 (2018).

35 van der Meer, A. J. et al. Systemic inflammation induces release of cell-free DNA from hematopoietic and parenchymal cells in mice and humans. Blood Adv 3, 724728, doi:10.1182/bloodadvances.2018018895 (2019).

36 Tsuji, N. \& Agbor-Enoh, S. Cell-free DNA beyond a biomarker for rejection: Biological trigger of tissue injury and potential therapeutics. $J$ Heart Lung Transplant 40, 405-413, doi:10.1016/j.healun.2021.03.007 (2021).

37 Lucas, C. et al. Longitudinal analyses reveal immunological misfiring in severe COVID-19. Nature 584, 463-469, doi:10.1038/s41586-020-2588-y (2020).

38 Organization, W. H. (2020).

39 Vanderbeke, L. et al. Monocyte-driven atypical cytokine storm and aberrant neutrophil activation as key mediators of COVID-19 disease severity. Nat Commun 12, 4117, doi:10.1038/s41467-021-24360-w (2021).

40 Xiao, N. et al. Integrated cytokine and metabolite analysis reveals immunometabolic reprogramming in COVID-19 patients with therapeutic implications. Nat Commun 12, 1618, doi:10.1038/s41467-021-21907-9 (2021). 
141 Manuel, O. \& Estabrook, M. RNA respiratory viral infections in solid organ

transplant recipients: Guidelines from the American Society of Transplantation Infectious Diseases Community of Practice. Clin Transplant 33, e13511, doi:10.1111/ctr.13511 (2019).

42 Gärtner, B. C. \& Avery, R. K. Respiratory viral infections in solid organ transplant recipients: New insights from multicenter data. Am J Transplant 21, 1685-1686, doi:10.1111/ajt.16408 (2021).

43 Paulsen, G. C. \& Danziger-Isakov, L. Respiratory Viral Infections in Solid Organ and Hematopoietic Stem Cell Transplantation. Clin Chest Med 38, 707-726, doi:10.1016/j.ccm.2017.07.012 (2017).

44 Goussous, N. et al. Donor-derived Cell-free DNA in Infections in Kidney Transplant Recipients: Case Series. Transplant Direct 6, e568, doi:10.1097/txd.0000000000001019 (2020).

45 Whitlam, J. B. et al. Diagnostic application of kidney allograft-derived absolute cellfree DNA levels during transplant dysfunction. Am J Transplant 19, 1037-1049, doi:10.1111/ajt.15142 (2019).

46 Ackermann, M. et al. Pulmonary Vascular Endothelialitis, Thrombosis, and Angiogenesis in Covid-19. $N$ Engl $J$ Med 383, 120-128, doi:10.1056/NEJMoa2015432 (2020).

47 Blanco-Melo, D. et al. Imbalanced Host Response to SARS-CoV-2 Drives Development of COVID-19. Cell 181, 1036-1045.e1039, doi:10.1016/j.cell.2020.04.026 (2020). 
148 Ramos-Casals, M., Brito-Zerón, P. \& Mariette, X. Systemic and organ-specific immune-related manifestations of COVID-19. Nat Rev Rheumatol 17, 315-332, doi:10.1038/s41584-021-00608-z (2021).

49 Tay, M. Z., Poh, C. M., Rénia, L., MacAry, P. A. \& Ng, L. F. P. The trinity of COVID19: immunity, inflammation and intervention. Nat Rev Immunol 20, 363-374, doi:10.1038/s41577-020-0311-8 (2020).

50 Avery, R. K. et al. Inpatient COVID-19 outcomes in solid organ transplant recipients compared to non-solid organ transplant patients: a retrospective cohort. American Journal of Transplantation 21, 2498-2508 (2021).

51 Stephanie, G. Y. et al. Early experience with COVID-19 and solid organ transplantation at a US high-volume transplant center. Transplantation 104, 2208 (2020).

52 Tschopp, J. et al. First experience of SARS-CoV-2 infections in solid organ transplant recipients in the Swiss Transplant Cohort Study. American Journal of Transplantation 20, 2876-2882 (2020).

53 Ranabothu, S. et al. Outcomes of COVID-19 in Solid Organ Transplants. Cureus 12, e11344, doi:10.7759/cureus.11344 (2020).

54 Verhoeven, J. G. et al. Liquid biopsies to monitor solid organ transplant function: a review of new biomarkers. Therapeutic drug monitoring 40, 515-525 (2018).

55 Nassiri, F. et al. Detection and discrimination of intracranial tumors using plasma cell-free DNA methylomes. Nat Med 26, 1044-1047, doi:10.1038/s41591-0200932-2 (2020). 
156 Scozzi, D. et al. Circulating mitochondrial DNA is an early indicator of severe illness and mortality from COVID-19. JCI Insight 6, doi:10.1172/jci.insight.143299 (2021).

57 Gould, T. J. et al. Cell-free DNA modulates clot structure and impairs fibrinolysis in sepsis. Arteriosclerosis, Thrombosis, and Vascular Biology 35, 2544-2553 (2015).

58 von Meijenfeldt, F. A. et al. Elevated plasma levels of cell-free DNA during liver transplantation are associated with activation of coagulation. Liver Transplantation 24, 1716-1725 (2018).

59 Wong, D. W. L. et al. Multisystemic Cellular Tropism of SARS-CoV-2 in Autopsies of COVID-19 Patients. Cells 10, doi:10.3390/cells10081900 (2021).

60 Bozzano, F. et al. Extensive activation, tissue trafficking, turnover and functional impairment of NK cells in COVID-19 patients at disease onset associates with subsequent disease severity. PLoS Pathog 17, e1009448, doi:10.1371/journal.ppat.1009448 (2021).

61 Huerga Encabo, H. et al. Human Erythroid Progenitors Are Directly Infected by SARS-CoV-2: Implications for Emerging Erythropoiesis in Severe COVID-19 Patients. Stem Cell Reports 16, 428-436, doi:10.1016/j.stemcr.2021.02.001 (2021).

62 Zuo, Y. et al. Neutrophil extracellular traps in COVID-19. JCI Insight 5, doi:10.1172/jci.insight.138999 (2020).

63 Veras, F. P. et al. SARS-CoV-2-triggered neutrophil extracellular traps mediate COVID-19 pathology. J Exp Med 217, doi:10.1084/jem.20201129 (2020).

64 Zuo, Y. et al. Neutrophil extracellular traps and thrombosis in COVID-19. J Thromb Thrombolysis, 1-8, doi:10.1007/s11239-020-02324-z (2020). 
165 van den Bosch, T. P., Kannegieter, N. M., Hesselink, D. A., Baan, C. C. \& Rowshani,

A. T. Targeting the Monocyte-Macrophage Lineage in Solid Organ Transplantation. Front Immunol 8, 153, doi:10.3389/fimmu.2017.00153 (2017).

66 Bernardes, J. P. et al. Longitudinal Multi-omics Analyses Identify Responses of Megakaryocytes, Erythroid Cells, and Plasmablasts as Hallmarks of Severe COVID19. Immunity 53, 1296-1314.e1299, doi:10.1016/j.immuni.2020.11.017 (2020).

67 Renner, K. et al. Severe T cell hyporeactivity in ventilated COVID-19 patients correlates with prolonged virus persistence and poor outcomes. Nat Commun 12, 3006, doi:10.1038/s41467-021-23334-2 (2021).

68 Mallavia, B. et al. Mitochondrial DNA stimulates TLR9-dependent neutrophil extracellular trap formation in primary graft dysfunction. American journal of respiratory cell and molecular biology 62, 364-372 (2020).

69 Scozzi, D. et al. Mitochondrial damage-associated molecular patterns released by lung transplants are associated with primary graft dysfunction. American Journal of Transplantation 19, 1464-1477 (2019).

70 Sperry, B. W., Khumri, T. M. \& Kao, A. C. Donor-derived cell-free DNA in a heart transplant patient with COVID-19. Clinical transplantation 34, e14070 (2020).

71 Bunnapradist, S. et al. Extremely High Cell-free DNA Levels Observed in Renal Allograft Patient With SARS-CoV-2 Infection. Transplantation Direct 7 (2021).

72 Grskovic, M. et al. Validation of a clinical-grade assay to measure donor-derived cell-free DNA in solid organ transplant recipients. The Journal of Molecular Diagnostics 18, 890-902 (2016). 
173 Wong, L., Scott, S., Grskovic, M., Dholakia, S. \& Robert, W. The Evolution and

2

3

474 Brown, J., Pirrung, M. \& McCue, L. A. FQC Dashboard: integrates FastQC results

775 Martin, M. Cutadapt removes adapter sequences from high-throughput

976 Krueger, F. \& Andrews, S. R. Bismark: a flexible aligner and methylation caller for

1177 Hansen, K. D., Langmead, B. \& Irizarry, R. A. BSmooth: from whole genome bisulfite sequencing reads to differentially methylated regions. Genome biology 13, R83 (2012).

78 Benjamini, Y. \& Hochberg, Y. Controlling the false discovery rate: a practical and powerful approach to multiple testing. Journal of the Royal statistical society: series B (Methodological) 57, 289-300 (1995). 
1 Table 1: Baseline demographic and clinical characteristics of the SOT patients with

2 COVID-19

\begin{tabular}{|c|c|c|c|c|c|}
\hline \multicolumn{2}{|c|}{ Variables } & All SOT & $\begin{array}{c}\text { Mild-moderate } \\
\text { sOT, n=32 } \\
(72.7 \%)\end{array}$ & $\begin{array}{l}\text { Severe SOT, } \\
n=12(27.3 \%)\end{array}$ & $P$ value \\
\hline \multicolumn{6}{|l|}{ Demographic } \\
\hline Age, years & median (IQR) & $\begin{array}{l}54.5(43.5- \\
66.5)\end{array}$ & $50(41.5-62.5)$ & $\begin{array}{l}62.5(53.25- \\
68.0)\end{array}$ & 0.030 \\
\hline \multirow{3}{*}{ Sex, n (\%) } & Male & 27 (61.4\%) & 18 (56.25\%) & 9 (75\%) & \\
\hline & Female & 17 (38.6\%) & $14(43.75 \%)$ & $3(25 \%)$ & 0.315 \\
\hline & Black & $16(36.4 \%)$ & $11(34.4 \%)$ & $5(41.7 \%)$ & \\
\hline \multirow{4}{*}{$\begin{array}{l}\text { Race/ethinicity, n } \\
\text { (\%) }\end{array}$} & White & $23(52.3 \%)$ & $16(50 \%)$ & $7(58.3 \%)$ & \\
\hline & Hispanic & $4(9 \%)$ & $4(12.5)$ & 0 & \\
\hline & Others, unknow & $1(2.3 \%)$ & $1(3.1 \%)$ & 0 & \\
\hline & & $28.3(24.2$ - & $28.15(23.1$ - & $31.4(26.7-$ & \\
\hline BMI $\left(\mathrm{kg} / \mathrm{m}^{2}\right)$ & Median (IQR) & 34.3) & 33.6) & 35.3) & 0.393 \\
\hline \multirow{5}{*}{ 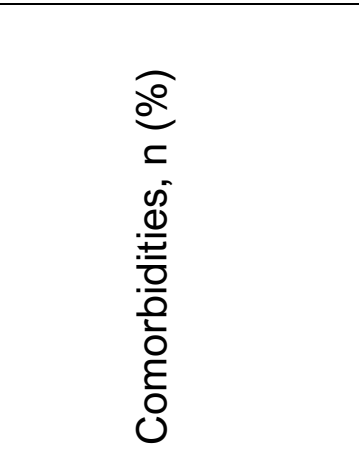 } & Obesity & $10(22.7 \%)$ & $6(18.6 \%)$ & $4(33.3 \%)$ & 0.422 \\
\hline & Diabetes & $21(47.7 \%)$ & 17 (53.1\%) & $4(33.3 \%)$ & 0.318 \\
\hline & HTN & 27 (61.4\%) & 20 (62.5\%) & 7 (58.3\%) & $>0.999$ \\
\hline & $\mathrm{CHF}$ & 7 (15.9\%) & $6(18.6 \%)$ & 1 (8.3\%) & 0.653 \\
\hline & CAD & 9 (20.5\%) & $6(18.6 \%)$ & $3(25 \%)$ & 0.687 \\
\hline
\end{tabular}




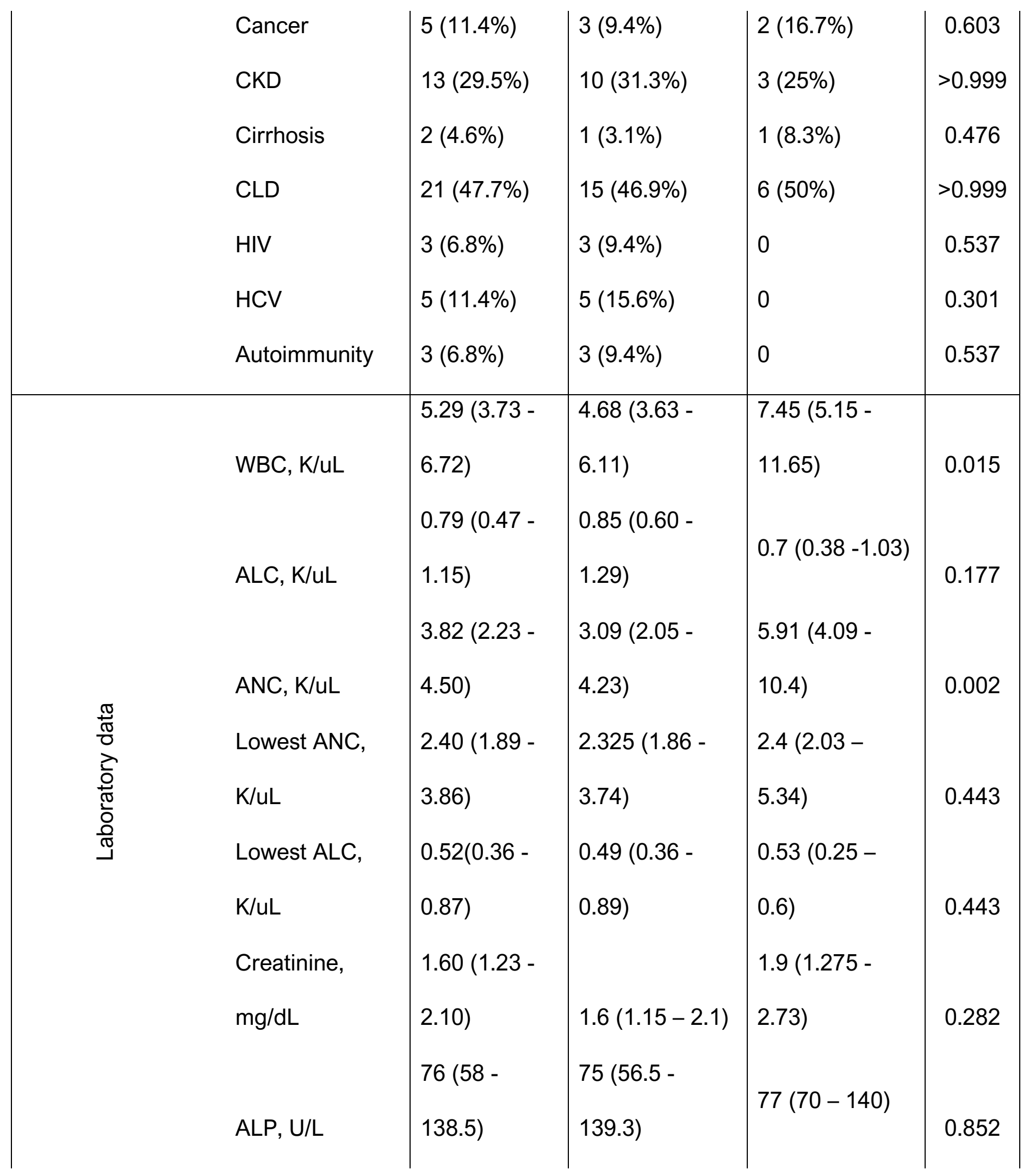




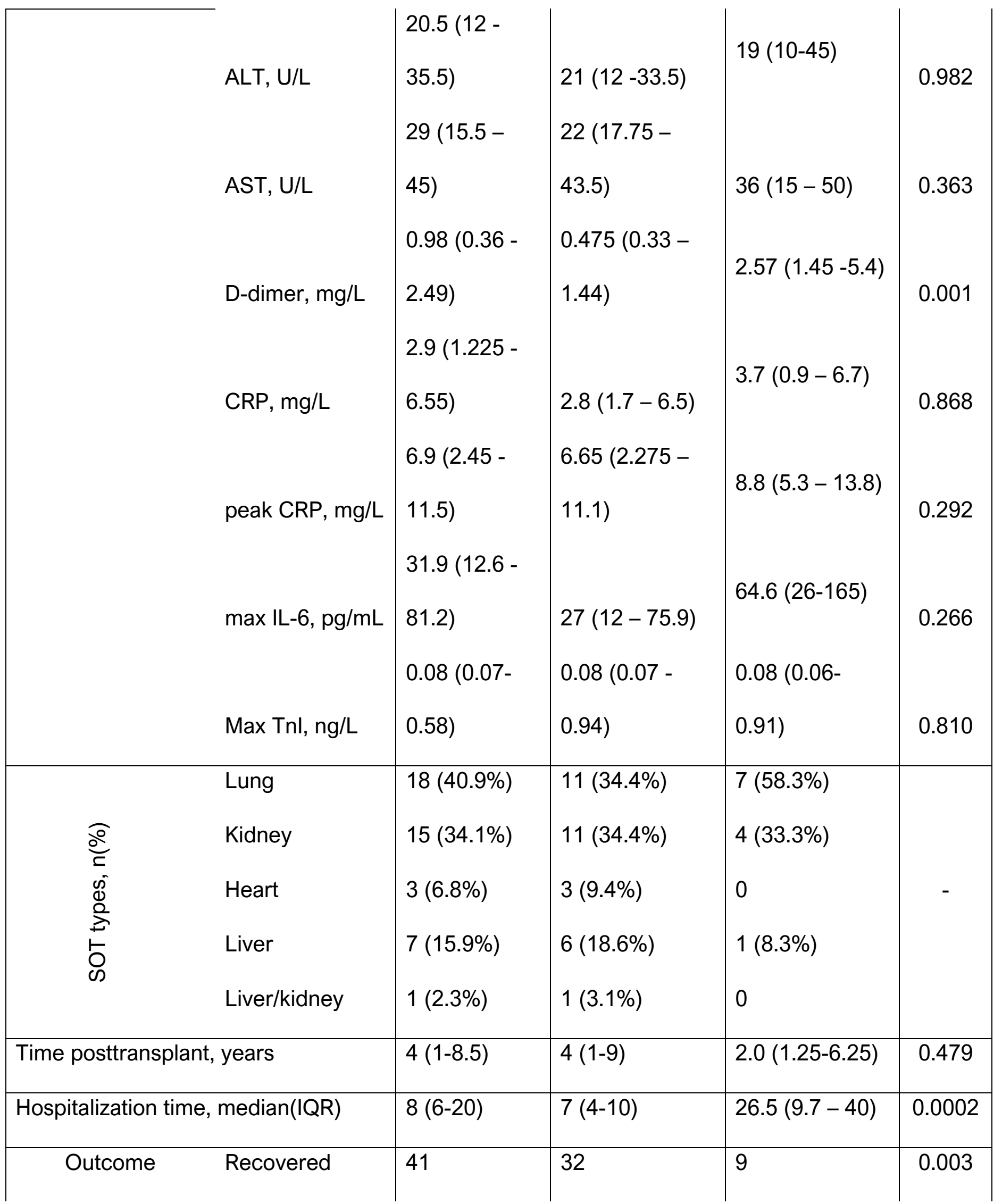




\begin{tabular}{|c|c|c|c|c|c|}
\hline & Deceased & 3 (25\%) & 0 & $3(25 \%)$ & \\
\hline $\begin{array}{c}\text { Pre-admission } \\
\text { MMF }\end{array}$ & $\begin{array}{l}\text { Yes } \\
\text { No }\end{array}$ & $\begin{array}{l}30 \\
13\end{array}$ & $\begin{array}{l}21 \\
10\end{array}$ & 9 & 0.727 \\
\hline MMF held & $\begin{array}{l}\text { Yes } \\
\text { No } \\
\text { Unknown }\end{array}$ & $\begin{array}{l}27 \\
3 \\
1\end{array}$ & $\begin{array}{l}20 \\
1 \\
1\end{array}$ & $\begin{array}{l}7 \\
2 \\
0\end{array}$ & 0.273 \\
\hline $\begin{array}{l}\text { COVID-19 } \\
\text { treatment }\end{array}$ & $\begin{array}{l}\text { RDV } \\
\text { Convalescent } \\
\text { plasma } \\
\text { Crizanlizumab } \\
\text { HCQ } \\
\text { Dex } \\
\text { Toci } \\
\text { None }\end{array}$ & $\begin{array}{l}23(52.7 \%) \\
20(47.3 \%) \\
2 \\
3 \\
4 \\
1 \\
3\end{array}$ & $\begin{array}{l}12 \\
16 \\
1 \\
3 \\
4 \\
0 \\
3\end{array}$ & $\begin{array}{l}1 \\
1 \\
0 \\
0 \\
1 \\
0\end{array}$ & $\begin{array}{c}0.002 \\
0.498 \\
- \\
- \\
- \\
- \\
-\end{array}$ \\
\hline \multicolumn{6}{|c|}{$\begin{array}{l}\text { Abbreviations: SOT, solid organ transplant; IQR, interquartile range; BMI, body mass index; HTN, } \\
\text { hypertension; CHF, congestive heart failure; CAD, coronary artery disease; CKD, chronic kidney } \\
\text { disease; CLD, chronic lung disease; HIV, Human immunodeficiency virus; HCV, Hepatitis C virus; } \\
\text { WBC, white blood cell count; ALC, absolute lymphocyte count; ANC, absolute neutrophil count; ALP, } \\
\text { alkaline phosphatase; ALT, alanine aminotransferase; AST, aspartate aminotransferase; CRP, C- } \\
\text { reactive protein; IL-6, Interleukin-6; TNI troponin I; RDV, remdesivir; HCQ, hydroxychloroquine; DEX, } \\
\text { dexamethasone; Toci, tocilizumab. }\end{array}$} \\
\hline
\end{tabular}


a)

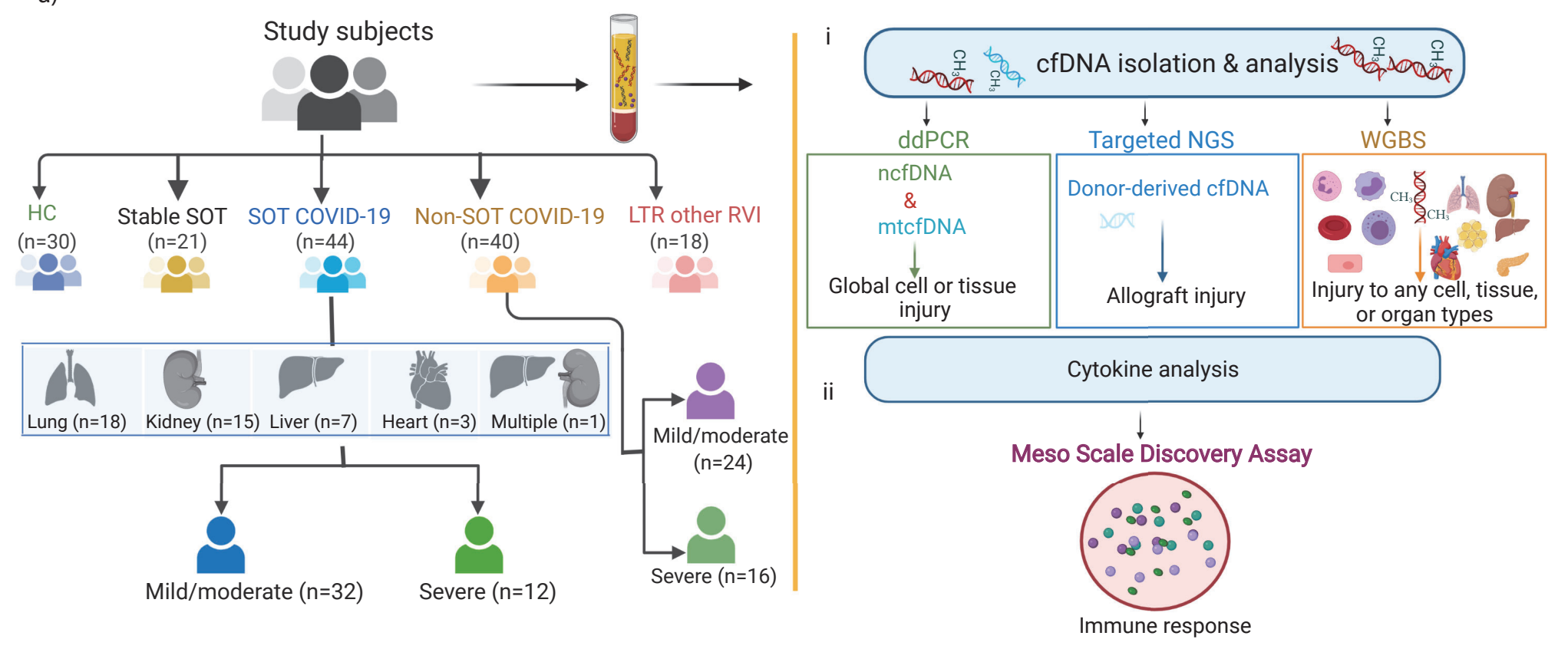

b)

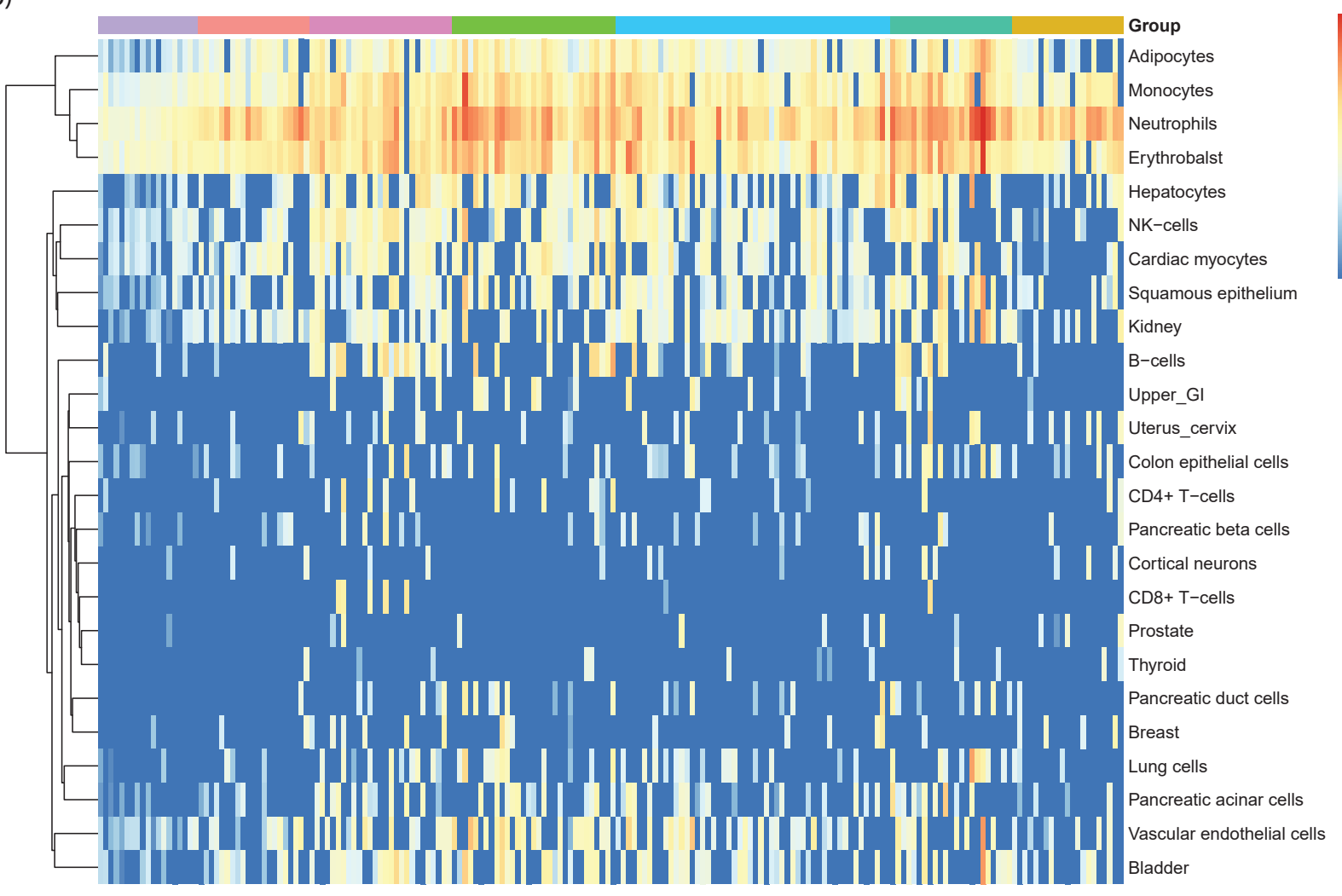

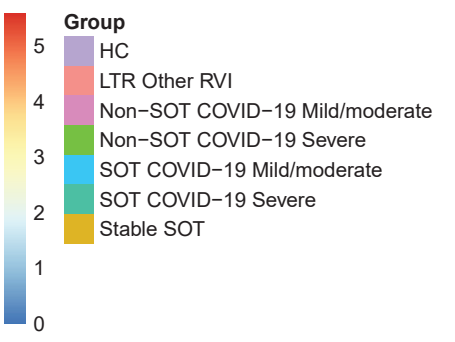


1 Figure 1. Study design and experimental workflow. a. Schematic diagram of study

2 subjects (including 30 Healthy controls [HC], 21 Stable solid organ transplant [SOT]

3 controls, 44 SOT COVID-19 [32 Mild/moderate and 12 severe] patients, 40 Non-SOT

4 COVID-19 [24 Mild/moderate and 16 severe] patients, and 18 Lung transplant recipients

5 with other respiratory viral infections [Other RVIs]). b. Schematic representation of plasma

6 cfDNA and cytokine quantification. c. Heatmap representation of tissue-specific cfDNA

7 profile across the groups. 

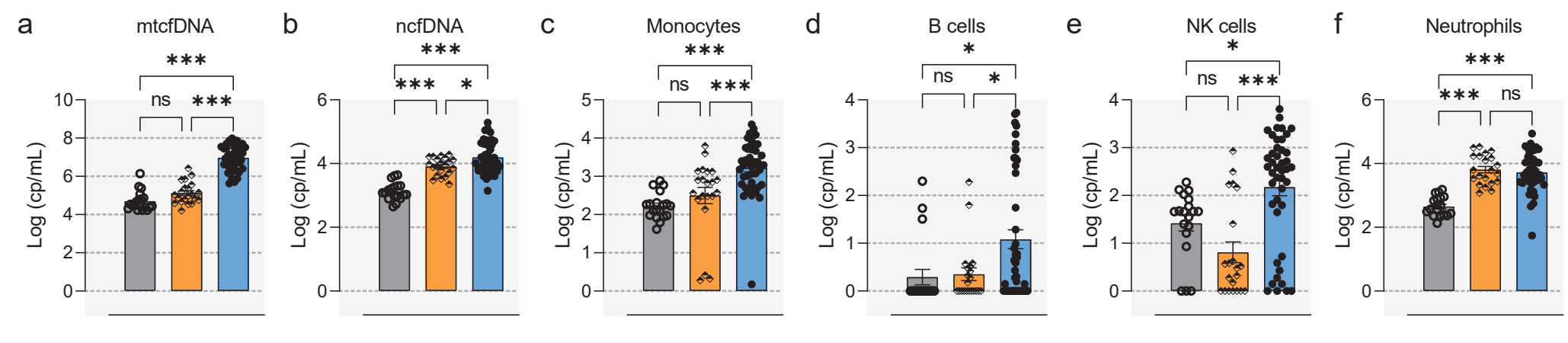

h Adipocytes
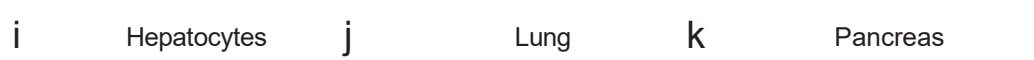

I Vascular endothelium
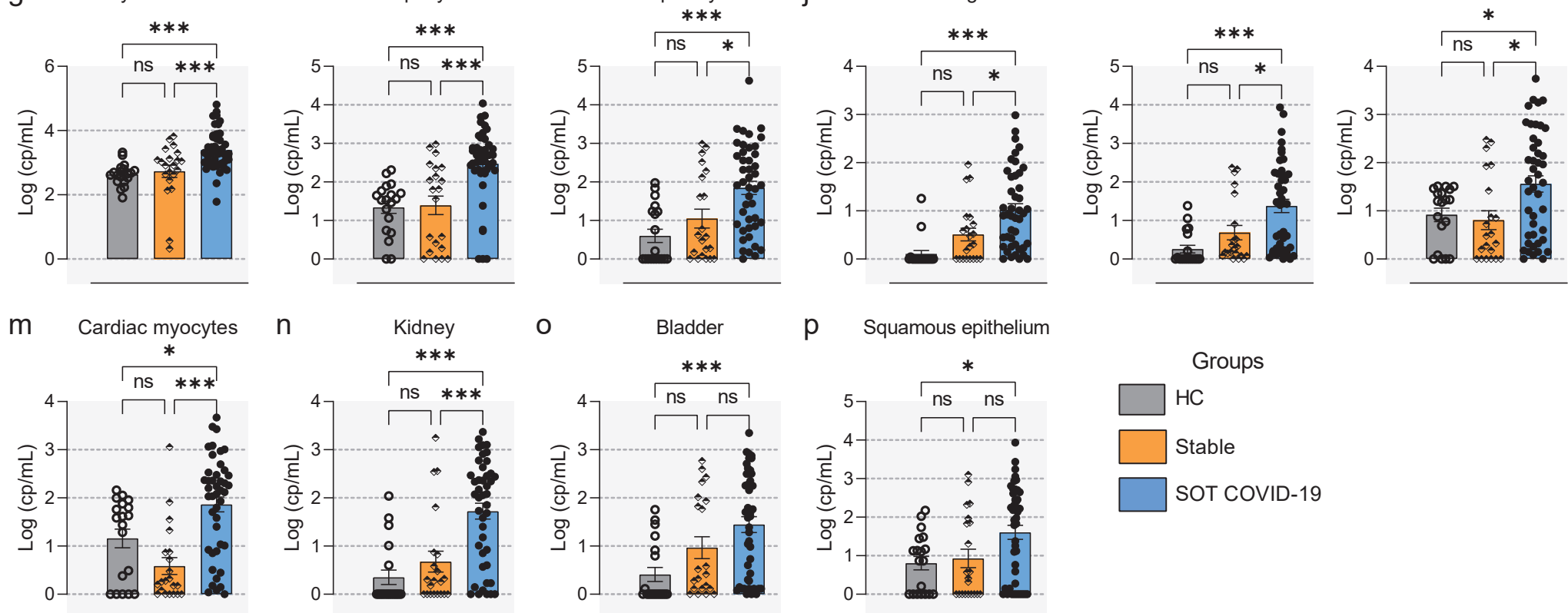

0

$p$

Squamous epithelium
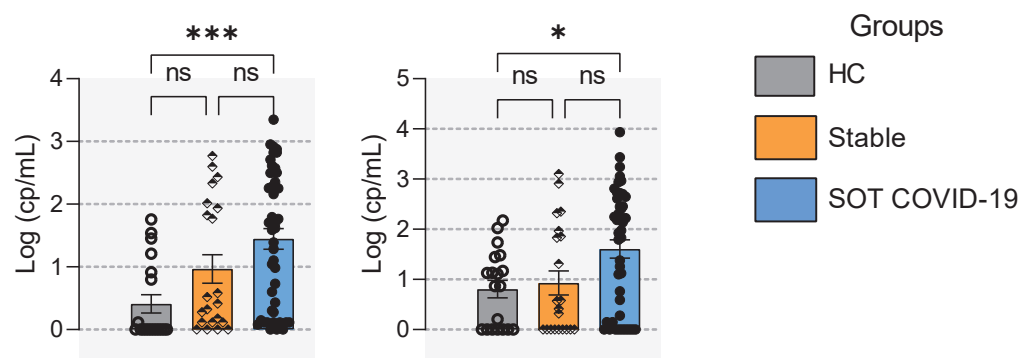
1 Figure 2. Elevation of circulating cfDNA levels in solid organ transplant recipients

2 (SOTRs) with COVID-19. Comparison of absolute plasma cell-free mitochondrial

3 (mtcfDNA) (a) and nuclear DNA (ncfDNA) (b) and tissue-specific cfDNA derived from

4 monocytes (c), B cells (d), NK cells (e), neutrophils (f), erythroblasts (g), vascular

5 endothelium (h), adipocytes (i), hepatocytes (j), lung (k), pancreas (I), cardiac myocytes

$6(\mathbf{m})$, kidney (n), bladder (o), and squamous epithelium (p) from Healthy controls (HCs)

$7 \quad(n=19)$, stable SOT controls $(n=21)$, and SOTRs with COVID-19 patients $(n=44)$. Plasma

8 ncfDNA and mtcfDNA concentrations were measured by digital droplet PCR. cfDNA

9 Whole-genome bisulfite sequencing was performed to measure tissue-specific cfDNA

10 profiles, leveraging tissue-specific DNA methylomes and deconvolution algorithms.

11 Median [interquartile range (IQR)] of cfDNA copies per $\mathrm{mL}(\mathrm{cp} / \mathrm{mL})$ are reported.

12 Statistical significance was determined by the Mann-Whitney test. Adjusted $p$ values are

13 reported (for multiple comparison and demographic factors (age, sex, and BMI). A p-value

$14 \leq 0.05$ and FDR $\leq 0.25$ was considered statistically significant;*: FDR $\leq 0.25$ and $p$-value $\leq$

$150.05,{ }^{* *}$ : FDR $\leq 0.1$ and $p$-value $\leq 0.05,{ }^{* * *}$ : FDR $\leq 0.05$ and $p$-value $\leq 0.05$, NS: FDR $>0.25$

16 or $p$-value $>0.05$. 

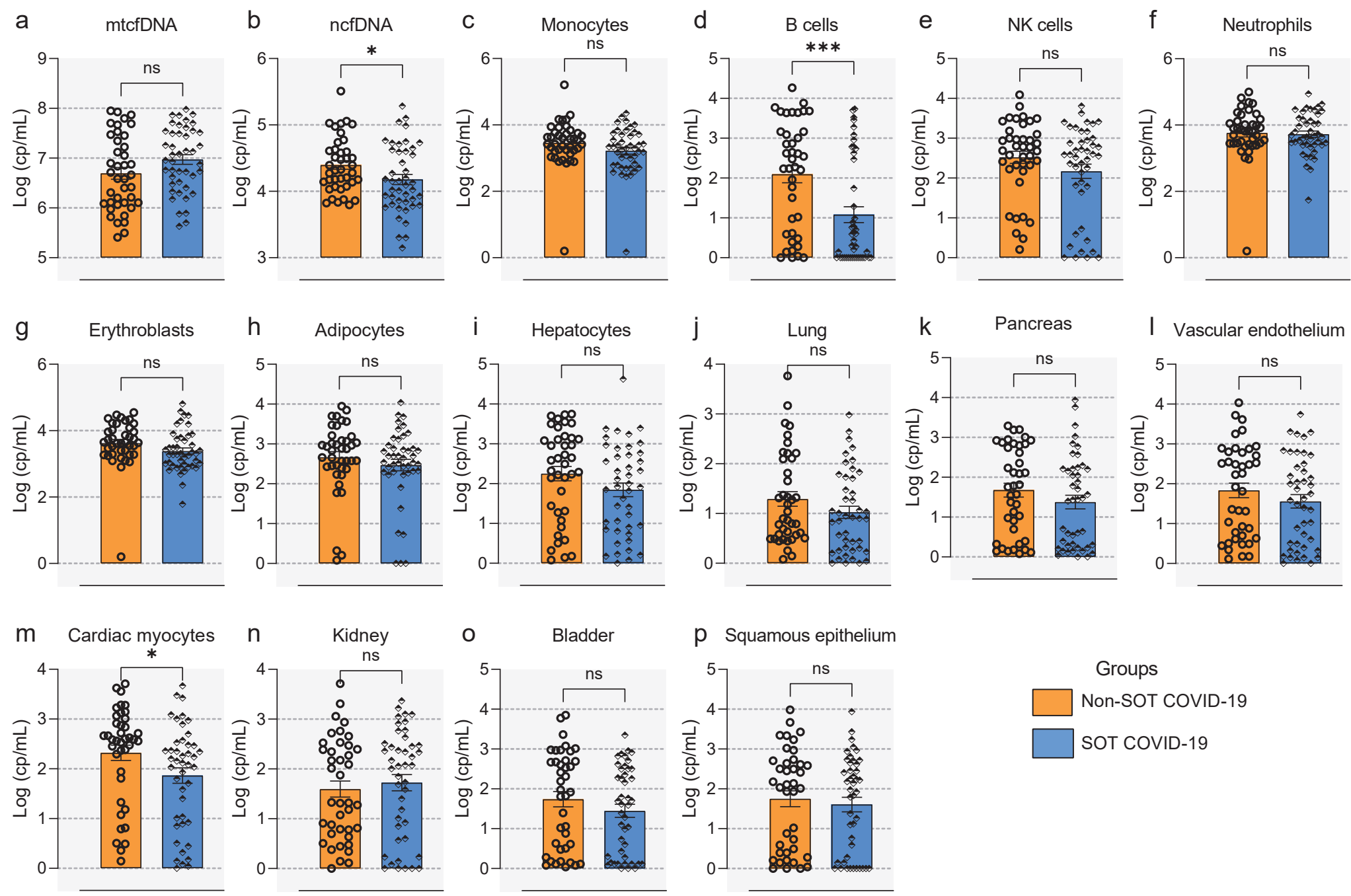

p Squamous epithelium

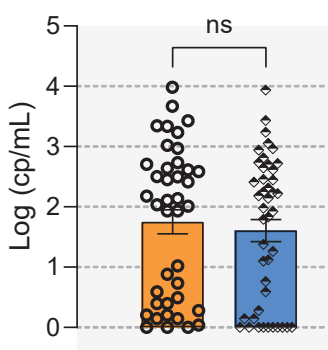

Groups

Non-SOT COVID-19

SOT COVID-19 
1 Figure 3. Comparable cfDNA profiles between SOT and Non-SOT COVID-19 patients.

2 Absolute plasma mtcfDNA (a) and ncfDNA (b) levels in Non-SOT ( $n=40)$ and SOT COVID-

319 patients. c-p. Quantification of tissue-specific cfDNA levels: monocytes (c), B cells (d),

4 NK cells (e), neutrophils (f), erythroblasts (g), vascular endothelium (h), adipocytes (i),

5 hepatocytes (j), lung (k), pancreas (I), cardiac myocytes ( $\mathbf{m})$, kidney $(\mathbf{n})$, bladder $(\mathbf{o})$, and

6 squamous epithelium (p) from Non-SOT and SOT COVID-19 patients. Statistical

7 significance was determined by the Mann-Whitney test. Adjusted $p$ values are reported

8 (for multiple comparison and demographic factors (age, sex, and BMI). A p-value $\leq 0.05$

9 and FDR $\leq 0.25$ was considered significant; ${ }^{*}$ FDR $\leq 0.25$ and p-value $\leq 0.05$, ${ }^{* *}$ : FDR $\leq 0.1$

10 and $p$-value $\leq 0.05,{ }^{* * *}$ : FDR $\leq 0.05$ and $p$-value $\leq 0.05$, NS: FDR $>0.25$ or $p$-value $>0.05$. 


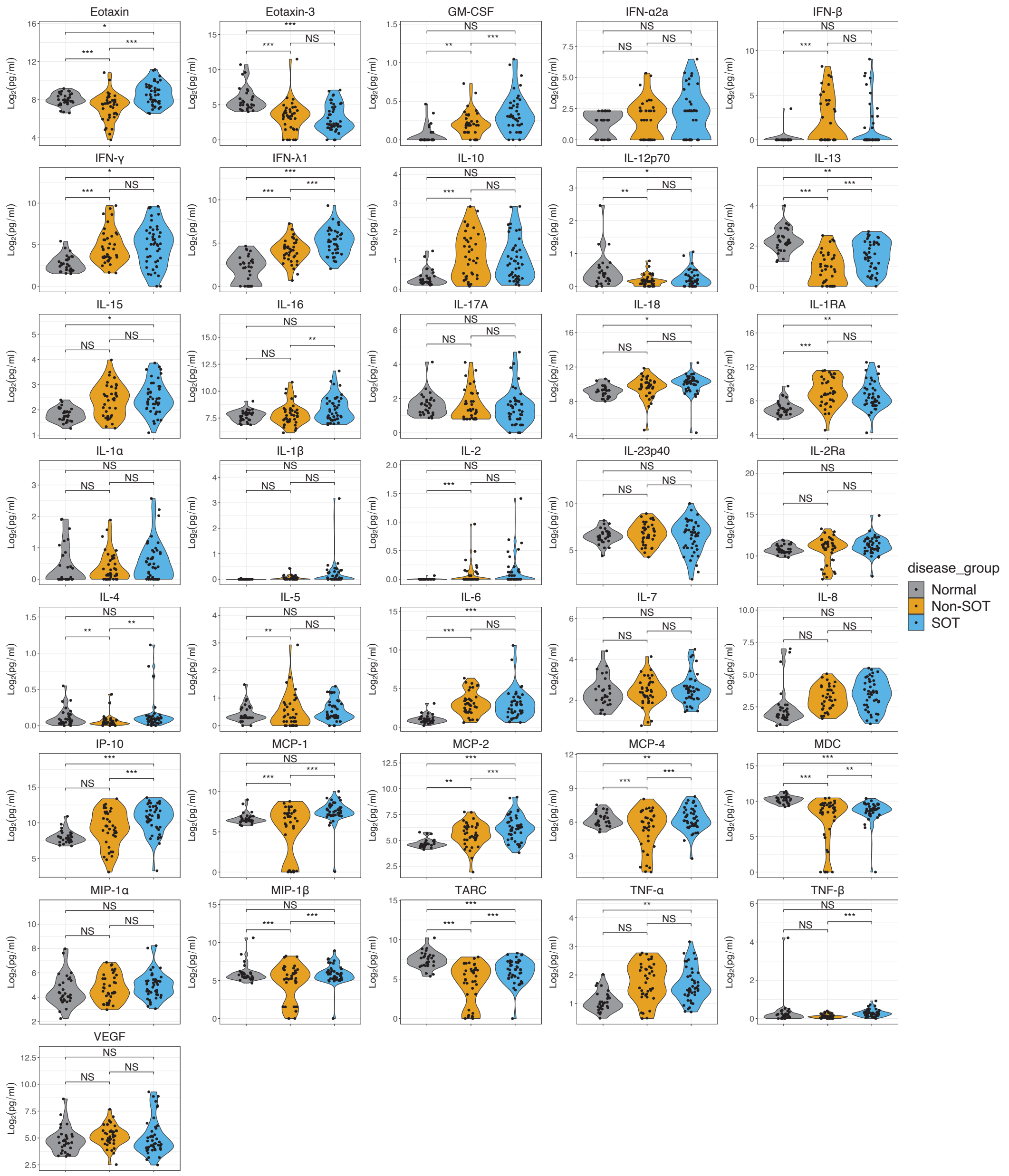


1 Figure 4. SOTRs with COVID-19 displayed dysregulated cytokine

2 responses. Comparison of plasma cytokine/chemokine levels on healthy controls $(n=30)$,

3 Non-SOT COVID-19 patients $(n=38)$ and SOTRs with COVID-19 $(n=44)$. Cytokine values

4 are reported as picograms per milliliter $(\mathrm{pg} / \mathrm{mL})$. Statistical significance was determined

5 by the Mann-Whitney test. Adjusted $p$ values are reported (for multiple comparison and

6 demographic factors (age, sex \& BMI). A p-value $\leq 0.05$ and FDR $\leq 0.25$ was considered

7 significant; $*$ FDR $\leq 0.25$ and $p$-value $\leq 0.05$, **: FDR $\leq 0.1$ and $p$-value $\leq 0.05$, ${ }^{* * *}$ : FDR $\leq$

80.05 and $p$-value $\leq 0.05$, NS: FDR $>0.25$ or $p$-value $>0.05$. 

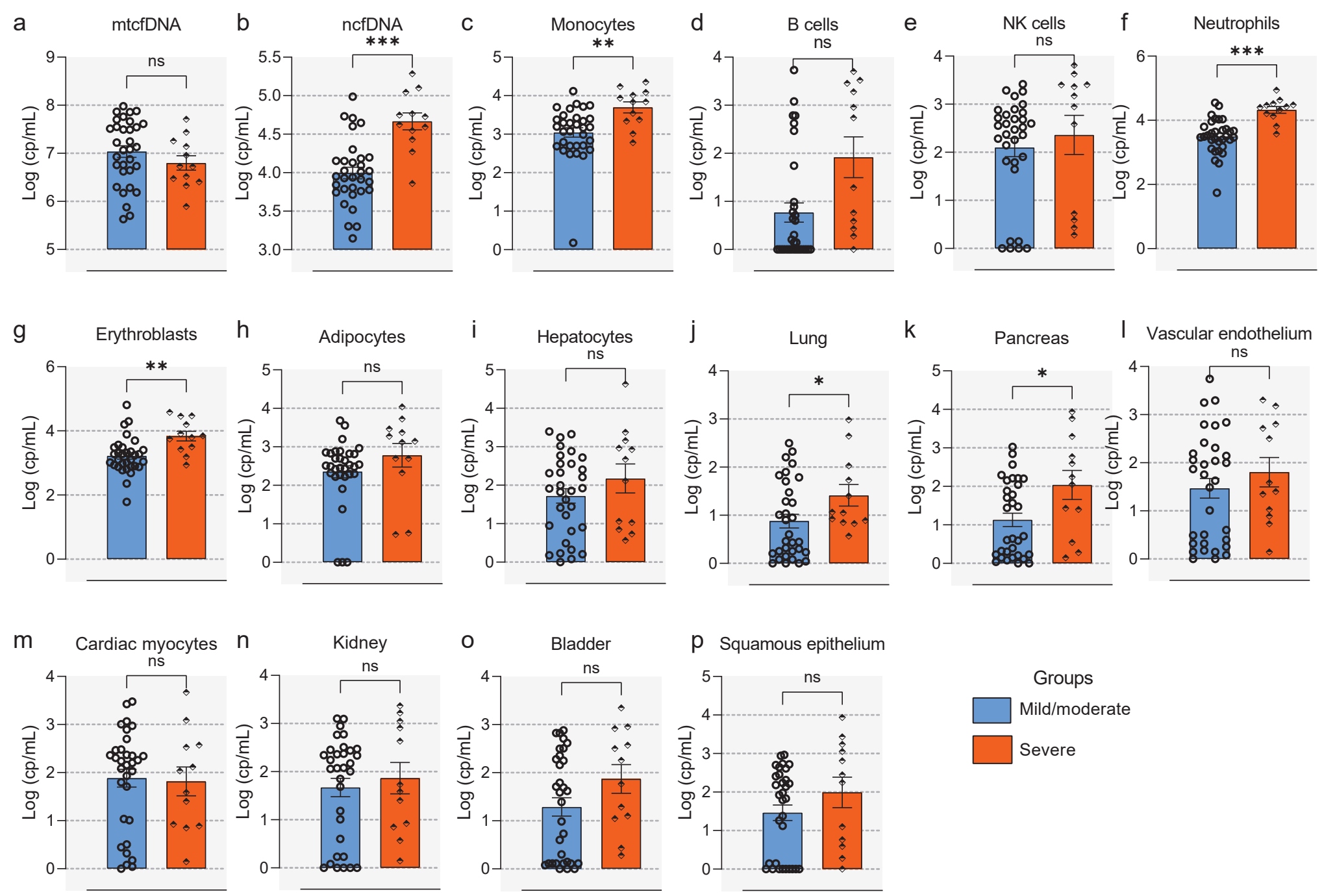

p Squamous epithelium

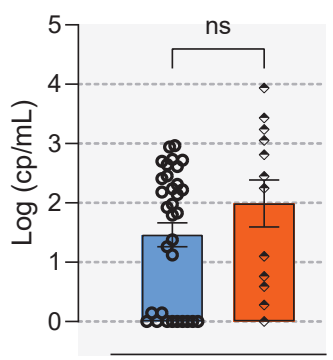

Groups

Mild/moderate

Severe

$\mathrm{n}$

cfDNA Features

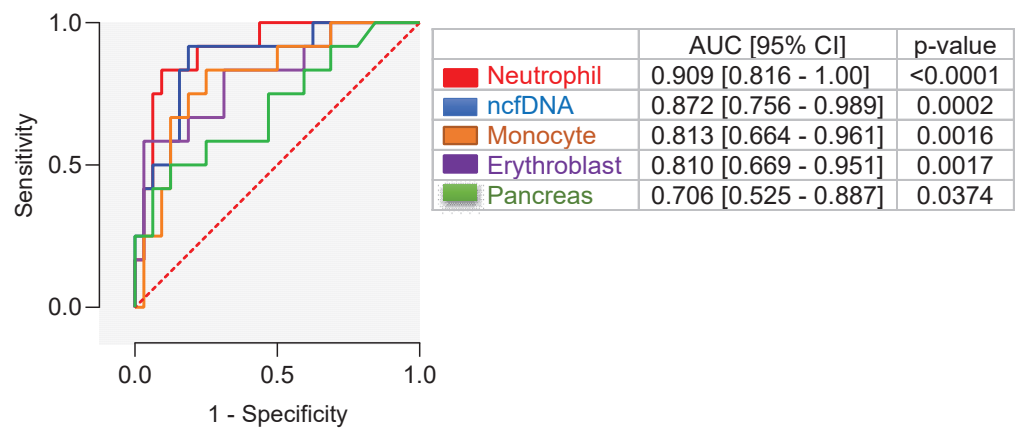


1 Figure 5. Plasma cfDNA features stratify SOT COVID-19 patients by severity. COVID-19

2 subjects were grouped based on disease severity as mild/moderate and severe.

3 Comparisons of absolute total mtcfDNA (a) ncfDNA and mtcfDNA (b) and tissue-specific

4 cfDNA levels derived monocytes (c), B cells (d), NK cells (e), neutrophils (f), erythroblasts

5 (g), vascular endothelium (h), adipocytes (i), hepatocytes (j), lung (k), pancreas (I),

6 cardiac myocytes $(\mathbf{m})$, kidney (n), bladder (o), and squamous epithelium (p) from SOTRs

7 with mild-moderate $(n=12)$ and severe disease $(n=32) .(q)$. ROC curve analyses using

8 admission cfDNA profile was performed to identify SOT patients with severe COVID-19.

9 Statistical significance was determined by the Mann-Whitney test. Adjusted $p$ values are

10 reported (for multiple comparison and demographic factors (age, sex, and

11 BMI). Statistical significance was determined by the Mann-Whitney test. A p-value $\leq 0.05$

12 and FDR $\leq 0.25$ was considered significant; ${ }^{*}$ FDR $\leq 0.25$ and $p$-value $\leq 0.05$, **: FDR $\leq 0.1$

13 and $p$-value $\leq 0.05,{ }^{* * *}$ : FDR $\leq 0.05$ and $p$-value $\leq 0.05$, NS: FDR $>0.25$ or $p$-value $>0.05$. 
1 Figure 6. Plasma cytokine levels associated with COVID-19 disease severity in SOTRs.

2 (a). Comparison of plasma cytokine/chemokine levels in mild/moderate $(n=32)$ verses

3 severe SOTRs with COVID-19 $(n=12)$. Cytokine values are reported as picograms per

4 milliliter (pg/mL). (b). ROC curve analyses using admission cytokine profile was performed

5 to identify SOT patients with severe COVID-19. Statistical significance was determined by

6 Mann-Whitney test. Adjusted $\mathrm{p}$ values are reported (for multiple comparison and

7 demographic factors (age, sex, and BMI). A p-value $\leq 0.05$ and FDR $\leq 0.25$ was considered

8 significant;: FDR $\leq 0.25$ and $p$-value $\leq 0.05$, **: FDR $\leq 0.1$ and $p$-value $\leq 0.05,{ }^{* * *}$ : FDR $\leq$

90.05 and $p$-value $\leq 0.05$, NS: FDR $>0.25$ or $p$-value $>0.05$. 

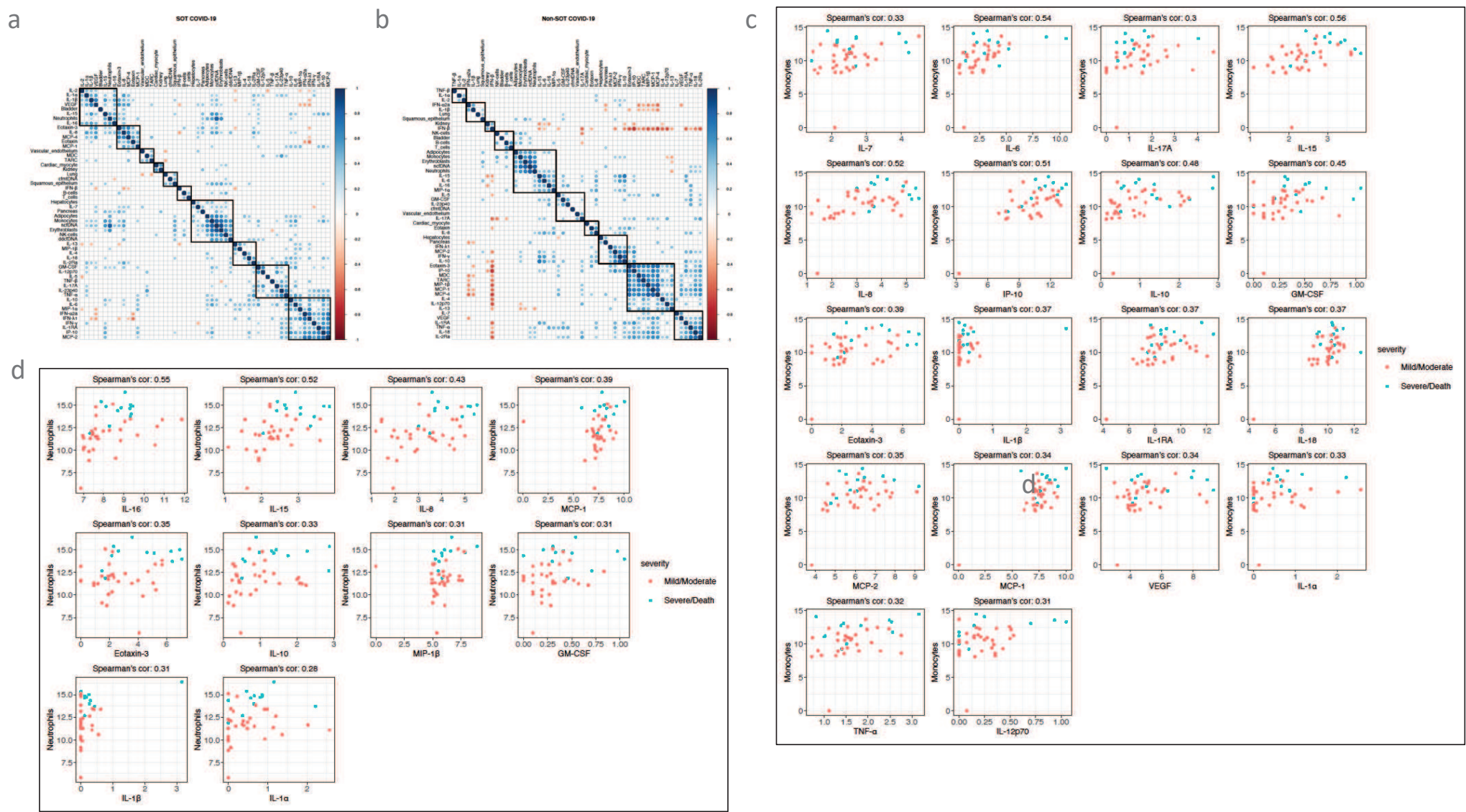
1 Figure 7. A strong association between circulating cfDNA and inflammatory

2 cytokine/chemokine signatures in SOTRs with COVID-19. Admission plasma cfDNA and

3 cytokine were measured from SOTRs with COVID-19 $(n=44)$ and Non-SOT COVID-19

4 patients $(n=38)$. Pearson correlation matrix analysis of cfDNA profiles and

5 cytokines/chemokines levels in SOTRs (a) with COVID-19 and Non-SOT COVID-19 (b).

6 Scatter Plot of the relationship between monocyte derived cfDNA and cytokines in SOT

7 patients with COVID-19 (c). Correlation scatter plot plots between neutrophil derived

8 cfDNA and cytokines in SOT patients with COVID-19 (d). Adjusted $p$ values are reported

9 (for multiple comparison and demographic factors (age, sex, and BMI). Statistical

10 significance was determined by the Spearman correlations test. A p-value $\leq 0.05$ and

11 FDR $\leq 0.25$ was considered significant;: FDR $\leq 0.25$ and $p$-value $\leq 0.05$, **: FDR $\leq 0.1$ and

$12 p$-value $\leq 0.05,{ }^{* * *}$ : FDR $\leq 0.05$ and $p$-value $\leq 0.05$, NS: FDR $>0.25$ or $p$-value $>0.05$. 

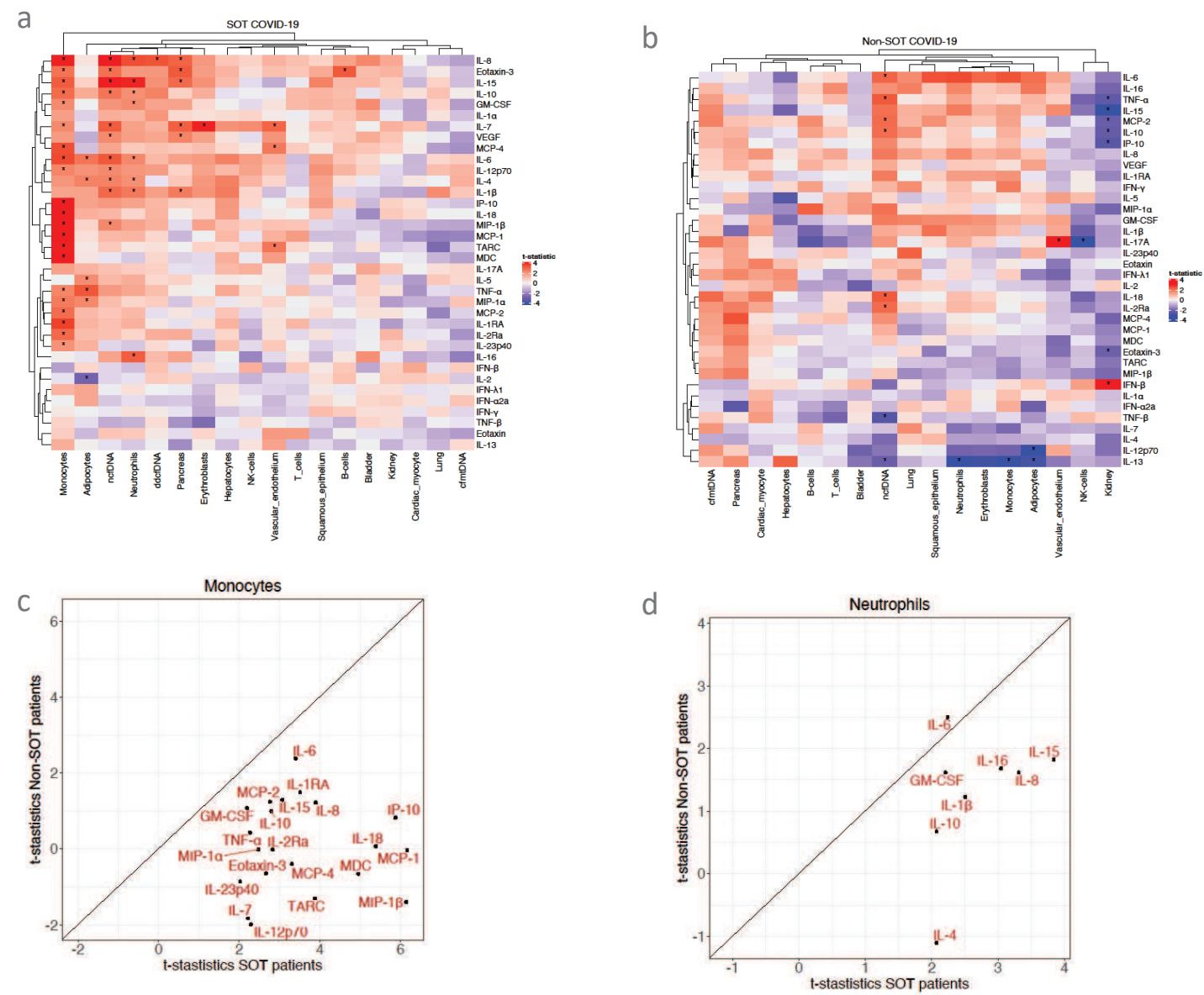
1 Figure 8. Linear regression analysis reveals an important association between cfDNA and

2 cytokine signatures. Hierarchical Clustering Heatmap of circulating cfDNA and cytokine

3 signatures in SOT (a) and Non-SOT patients COVID-19 (b). t-statistics scatterplot plots

4 between monocyte derived cfDNA and cytokines in SOT patients with COVID-19 (c). t-

5 statistics scatter plot plots between neutrophil derived cfDNA and cytokines in SOT

6 patients with COVID-19 (d). Linear regression analysis between cfDNA features and

7 cytokine profile was conducted. Adjusted $p$ values are reported (for multiple comparison

8 and demographic factors (age, sex, and BMI). A p-value $\leq 0.05$ and FDR $\leq 0.25$ was

9 considered significant; : FDR $\leq 0.25$ and $p$-value $\leq 0.05$, **: FDR $\leq 0.1$ and $p$-value $\leq 0.05$,

$10 * * *$ FDR $\leq 0.05$ and $p$-value $\leq 0.05$, NS: FDR $>0.25$ or $p$-value $>0.05$ 

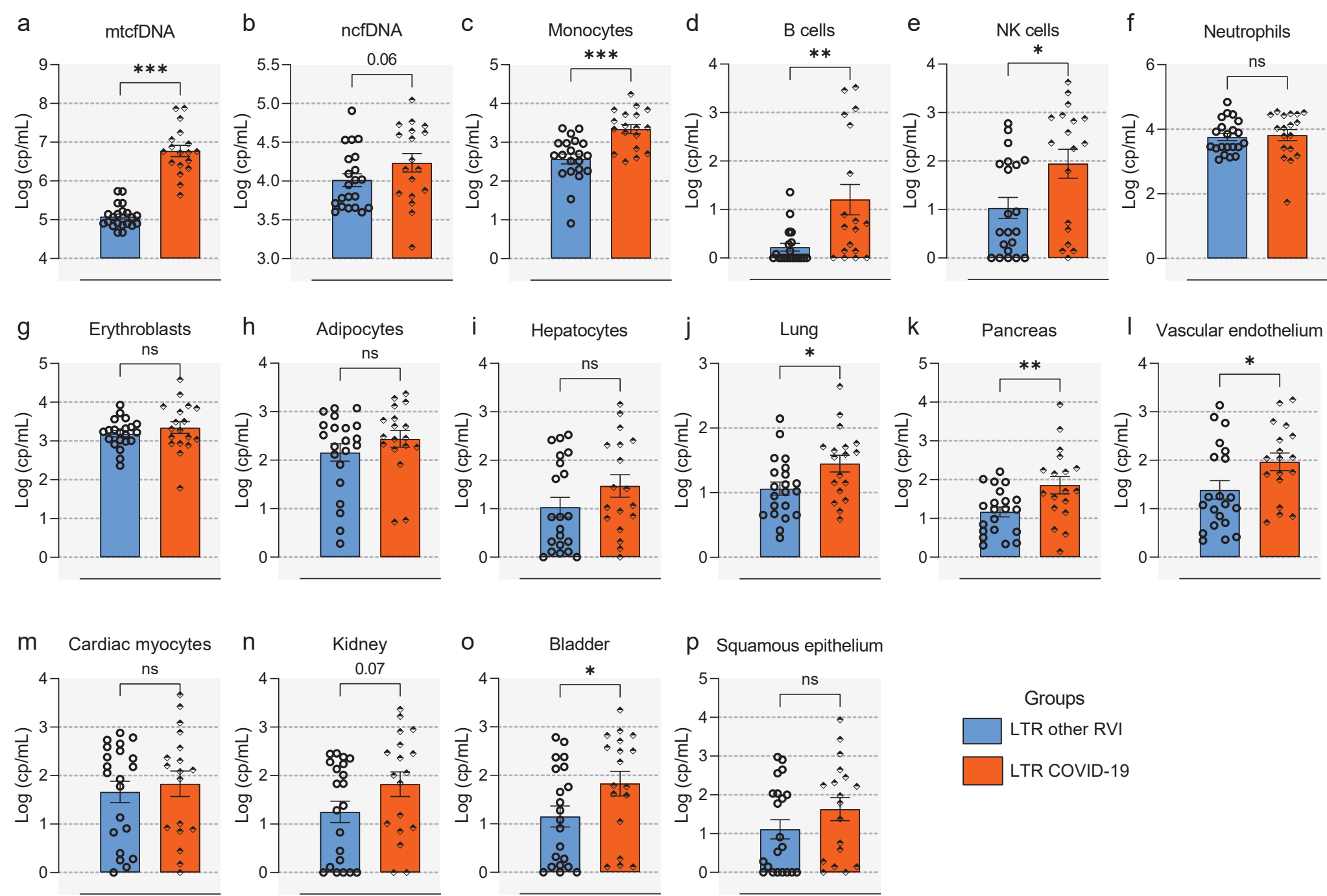

p Squamous epithelium

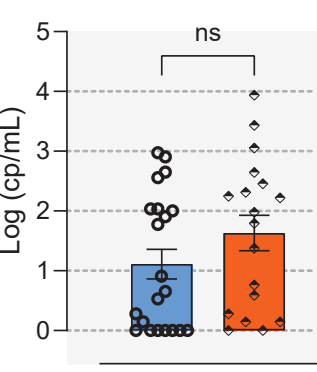

Groups

LTR other RVI

LTR COVID-19 
1 Figure 9. High plasma cfDNA levels in lung transplant recipients with COVID-19

2 compared to other respiratory viruses. Comparisons of absolute total mtcfDNA (a)

3 ncfDNA and mtcfDNA (b) and tissue-specific cfDNA levels derived monocytes (c), B cells

4 (d), NK cells (e), neutrophils (f), erythroblasts (g), vascular endothelium (h), adipocytes

5 (i), hepatocytes (j), lung (k), pancreas (I), cardiac myocytes (m), kidney (n), bladder (o),

6 and squamous epithelium (p) among lung transplant recipients with COVID-19 ( $n=18)$ and

7 other respiratory viral infections (other RVIs) $(n=21)$. Statistical significance was

8 determined by the Mann-Whitney test. Adjusted $p$ values are reported (for multiple

9 comparison and demographic factors (age, sex \& BMI). A p-value $\leq 0.05$ and FDR $\leq 0.25$

10 was considered statistically significant; * FDR $\leq 0.25$ and $p$-value $\leq 0.05$, **. FDR $\leq 0.1$ and

$11 p$-value $\leq 0.05,{ }^{* * *}$ : FDR $\leq 0.05$ and $p$-value $\leq 0.05$, NS: FDR $>0.25$ or $p$-value $>0.05$. 

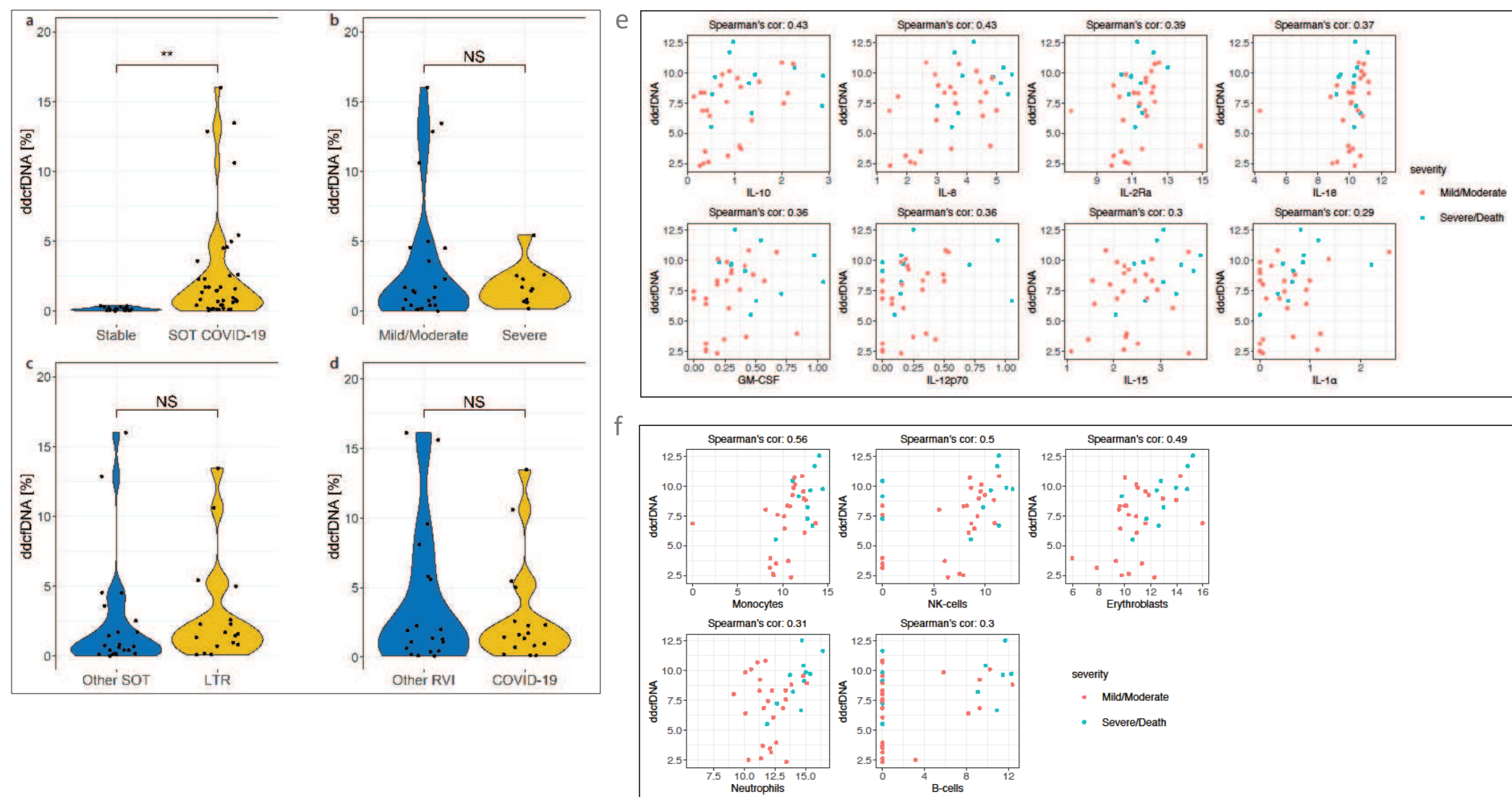

severity

- Mild/Moderate

- Severe/Death 
1 Figure 10. Elevated ddcfDNA level in SOTRs with COVID-19. (a) comparison of ddcfDNA

2 level between stable transplant recipients $(n=18)$ and SOTRs with COVID-19 $(n=39) .(a)$

3 comparison of ddcfDNA level between stable controls $(n=21)$ and SOTRs with COVID-19

$4 \quad(n=44)$. (b) comparison of ddcfDNA in SOT patients with mild/moderate $(n=28)$ and severe

5 COVID-19 disease $(n=12)$. (c) comparison of ddcfDNA level in lung transplant recipients

6 with COVID-19 (LTR) ( $n=17)$ and other SOT types $(n=22)$. (c) comparison of ddcfDNA

7 level in lung transplant recipients with COVID-19 (LTR) $(n=17)$ and other RVIs $(n=19)$. (d)

8 Correlation scatter plot plots between ddcfDNA and cytokines in SOT patients with

9 COVID-19. (e) Correlation scatter plot plots between ddcfDNA level and total and tissue-

10 specific cfDNA profiles in SOT patients with COVID-19. Statistical significance was

11 determined by Mann-Whitney test and Spearman correlations test. Adjusted $p$ values are

12 reported (for multiple comparison and demographic factors (age, sex, and BMI). A p-

13 value $\leq 0.05$ and FDR $\leq 0.25$ was considered statistically significant; ${ }^{*}$ FDR $\leq 0.25$ and $p$ -

14 value $\leq 0.05,{ }^{* *}$ : FDR $\leq 0.1$ and $p$-value $\leq 0.05,{ }^{* * *}$ : FDR $\leq 0.05$ and $p$-value $\leq 0.05$, NS:

15 FDR $>0.25$ or $p$-value $>0.05$. 
Andargie et al. 


\section{Additional Results}

2 1.1. Random forest analysis of cfDNA and cytokine signatures identified SOT patients with severe disease.

4 To characterize the important cfDNA features that distinguished SOT COVID-19 patients 5 needing ICU care, we performed random forest (RF) analysis (Suppl.Fig.2a). The top five cfDNA features at admission that differentiate SOT COVID-19 patients who eventually

7 develop severe disease from those with mild/moderate disease were ncfDNA, neutrophil, 8 monocyte, adipocyte, and erythroblast, respectively. We next performed RF analysis to 9 pinpoint important cytokine features that may affect disease severity (Suppl.Fig.2b). The 10 top five cytokine features that differentiate SOTRs with severe COVID-19 disease were

11 IL-15, IL-16, IL-23p40, TNF- $\alpha$, and IL-6. The cfDNA RF model showed good performance 12 to discriminate SOT COVID-19 patients into mild/moderate and severe groups, with an 13 area under the curve $(\mathrm{AUC})$ value of $0.815(95 \% \mathrm{Cl}=0.648-0.982)$. Additionally, the $\mathrm{RF}$

14 model for cytokine features also showed good performance (AUC $=0.796$ (95\% Cl: $0.643-$ 0.948) to distinguish SOT patients who develop severe disease versus mild/moderate.

16 Interestingly, combining the top five cfDNA and cytokine features in the RF analysis 17 improved the performance (AUC $=0.875(95 \% \mathrm{Cl}: 0.765-0.985)$ to identify SOT patients who progressed to severe COVID-19 (Suppl.Fig.2c and d). These results demonstrate

19 that integrated cfDNA and cytokine analysis improve the performance of RF model to 20 identify SOTRs who subsequently developed severe disease/died. 
1.2. cfDNA levels were comparable between lung and other SOT patients.

2 Given that lung transplant patients have the shortest post-transplant survival of all SOTRs

3 and that respiratory epithelium is the primary site for SARS-CoV-2 infection, we analyzed

4 and compared cfDNA profiles in LTRs with COVID-19 $(n=18,41 \%)$ and other SOT types

$5 \quad(n=26,59 \%)$. Our data showed no significant difference in total plasma cfDNA levels i.e.,

6 mt-cfDNA and ncfDNA, between LTRs with COVID-19 and those with other SOT types

$7 \quad(p>0.05$ and FDR>0.25; Suppl.Fig.6a and b). Comparing the cfDNA tissues-of-origin

8 profile, there were no significant differences in the plasma cfDNA levels derived from

9 hematopoietic cells and nonhematopoietic tissue types, including lung, adipocytes, heart

10 and kidney ( $p>0.05$ and FDR>0.25; Suppl.Fig.6c-p). This adds to the hypothesis that

11 COVID-19 is truly is a systemic disease in SOTRs.

12 1.3. Correlation of cfDNA profiles and cytokine signatures based on disease severity.

13 We calculated Spearman's correlation between all biomarkers pairs for patients in 14 different disease severity groups (Suppl.Fig.4). We observed three clusters in both mild 15 SOT and Non-SOT COVID-19 patients; however, the size and components of the clusters 16 are different, with the largest cluster that contains 12 cytokines in Non-SOT patients with 17 mild disease. In patients with severe disease, the correlation matrix showed more 18 fragmented correlation patterns, highlighting dysregulated cross-talk between cells and 19 cytokine levels. Thus, the differences in number, size, and components of clusters implied 20 distinct tissue injury and cytokine patterns between SOTRs with COVID-19 and Non-SOT 21 COVID-19 patients, as well as disease severity. Likewise, a two-way hierarchical cluster 22 heatmap generated showed divergent tissue injury patterns and cytokine response 
1 correlated with disease severity in both SOT and Non-SOT COVID-19 patients

2 (Suppl.Fig.5). The Spearman's correlation also revealed that cfDNA profiles were

3 correlated with altered cytokine levels both in mild/moderate and severe SOT patients,

4 including IL-8, IL-18, TNF- $\alpha$, IL-12p70, and IL-2Ra. In mild/moderate SOT patients,

5 monocyte-derived cfDNA correlated with multiple cytokines (IL-6, IL-8, IL-18, IL-10, IP-10,

6 IL-4, IL-15, IL-17A, GM-CSF, and MCP-2). Whereas in severe SOT patients adipocyte-

7 derived cfDNA showed the most pronounced association with cytokines (IL-5, TNF- $\alpha$,

8 Eotaxin, MIP-1a, MIP-1 $\beta$, IL-23p40, and IL-17A) followed by lung (MCP-1, IL-2Ra, and IL-

9 18) and monocytes (MDC, IL-12p70, and IL-23p40). Interestingly, the proinflammatory

10 cytokine/chemokine IL-18 and IL-8 were positively correlated with mtcfDNA (a DAMP) in

11 SOT COVID-19 patients with severe disease. Moreover, SOT patients with severe disease

12 displayed a strong correlation between cytokine levels and non-hematopoietic tissue-

13 derived cfDNA. Conversely, in Non-SOT patients with COVID-19, fewer interactions were

14 observed between cytokine levels and cfDNA profile, including in patients with severe

15 COVID-19 disease. Overall, these observations provide evidence of excessive cfDNA and exacerbated cytokine response interaction in SOTRs with COVID-19.

\subsection{Association between cfDNA profiles and known clinical and biochemical markers.}

We further investigated the association of cfDNA levels with clinical parameters and

19 visualized it as a heatmap (Supp.Fig.8). Unsupervised correlation clustering analysis revealed that D-dimer was co-clustered with total (ncfDNA) and tissue-specific cfDNA

21 levels (neutrophils, adipocytes, monocytes, ddcfDNA, NK-cells, and erythroblasts) and

22 liver function test enzymes (ALT and AST) with hepatocyte-derived cfDNA. We then 
assess how the tissue injury pattern relates to the age of the patients, a key determinant

2 of poor COVID-19 outcomes. Our analysis demonstrated that age was positively

3 correlated with total nuclear-derived cfDNA $(r=0.38, p=0.010)$ and tissue-specific cfDNA

4 derived from monocytes $(r=0.34, p=0.026)$, erythroblasts $(r=0.35, p=0.019)$, adipocytes

$5(r=0.30, p=0.046)$, pancreas $(r=0.34, p=0.025)$, ddcfDNA $(r=0.33, p=0.043)$ and marginally

6 correlated with cfDNA derived from neutrophil $(r=0.27, p=0.077)$. These results indicate

7 that cfDNA levels were influenced by age and the increased cfDNA levels were more

8 pronounced in older SOT COVID-19 patients. Total nuclear-origin and tissue-specific

9 cfDNA were not associated with sex in our cohort. Hospitalization length of stay was also

10 significantly associated with global nuclear $(r=0.44, p=0.003)$ and tissue-specific cfDNA

11 levels derived from monocyte $(r=0.38, p=0.012)$, neutrophil $(r=0.45, p=0.002)$, pancreas

$12(r=0.37, p=0.019)$ and ddcfDNA $(r=0.42, p=0.011)$.

14 Correlation analysis also revealed that cfDNA levels were correlated with clinically

15 established markers ( $p<0.05$ and FDR<0.25), as shown between neutrophil-derived cfDNA and ANC ( $r=0.45)$, NK cells-derived cfDNA and ALC ( $r=0.38)$, and hepatocyte-

17 derived cfDNA and liver function tests i.e., AST ( $r=0.70)$ and ALT ( $r=0.64)$. The serum D-

18 dimer level, an indirect marker of fibrinolysis and fibrin turnover, was strongly correlated with circulating ncfDNA ( $r=0.69)$ and cfDNA derived from monocyte $(r=0.51)$, neutrophil $20(r=0.63)$, erythroblast $(r=0.53)$, adipocyte $(r=0.54)$, pancreas $(r=0.33)$ and ddcfDNA 21 ( $r=0.38)$. We also observed peak levels of CRP, a marker of inflammation, were 22 significantly correlated with admission circulating level of ncfDNA $(r=0.33)$, hepatocyte- 
1 derived cfDNA ( $r=0.41)$ and ddcfDNA ( $r=0.38)$. Likewise, peak levels of IL-6 were

2 significantly associated ncfDNA ( $r=0.39)$ and tissue-specific cfDNA released from NK cell

3 ( $r=0.36)$, hepatocyte $(r=0.50)$, pancreas $(r=0.53)$, squamous epithelium $(r=0.32)$, and

4 kidney $(r=0.55)$. Overall, these results indicate the potential utility of cfDNA as a

5 comprehensive noninvasive "liquid biopsy" in transplant patients with COVID-19.

6 1.5. Longitudinal cfDNA and cytokine profiles in SOTRs with COVID-19.

7 To better understand the kinetics cfDNA profiles and cytokine levels, a subset of 5 SOT

8 COVID-19 (4 with mild/moderate infection and 1 with severe infection who died) and 4

9 Non-SOT COVID-19 patients underwent longitudinal blood sample collection (Fig.11). We

10 analyzed absolute tissue-specific cfDNA composition and cytokine profile kinetics. In a

11 LTR COVID-19 patient who eventually died, baseline plasma ncfDNA, ddcfdNA, mtcfDNA

12 cfDNA level were $39,216 \mathrm{cp} / \mathrm{mL}, 1.44 \%$, and $78,5248 \mathrm{cp} / \mathrm{mL}$, respectively. On day 3 after

13 admission, the plasma ncfDNA and mtcfDNA increased approximately 5 and 20 fold

14 compared to day 1 , respectively. The ncfDNA continued to increase from day 3 to day 7 ,

15 and the levels of the total cfDNA remained elevated above the median cfDNA levels for

16 severe patients until the patient died. In contrast, the total and tissue-specific cfDNA levels

17 gradually decreased with time in SOT patients with mild/moderate disease who eventually

18 recovered. Similarly, proinflammatory cytokines were markedly increased over time in

19 patients who eventually died. These findings suggest that SOT recipients, like Non-SOT

20 patients (Suppl.Fig.7), release excessive cfDNA amounts and display an exuberant

21 inflammatory response following SARS-CoV2 infection ${ }^{27,31,32}$. 
2 Suppl Table 1: Comparison of SOT and Non-SOT COVID-19 patients.

\begin{tabular}{|c|c|c|c|c|}
\hline \multicolumn{2}{|c|}{ Variables } & Non-SOT, n=40 & SOT, $n=44$ & $P$ value \\
\hline \multicolumn{5}{|l|}{ Demographic } \\
\hline & & $59.5(39.25$ - & $54.5(43.5$ - & \\
\hline Age, years & Median (IQR) & 70.5) & $66.5)$ & 0.651 \\
\hline \multirow{4}{*}{ Sex, n (\%) } & Male & $20(50 \%)$ & 27 (61.4\%) & 0.380 \\
\hline & Female & $20(50 \%)$ & 17 (38.6\%) & \\
\hline & Black/African & & & \\
\hline & American & $18(45 \%)$ & $16(36.4 \%)$ & 0.001 \\
\hline \multirow[t]{4}{*}{ Race/ethinicity, n (\%) } & White & $6(15 \%)$ & $23(52.3 \%)$ & \\
\hline & Hispanic & $11(27.5 \%)$ & $4(9 \%)$ & \\
\hline & Others, unknown & $5(12.5 \%)$ & $1(2.3 \%)$ & \\
\hline & & & $28.3(24.2-$ & \\
\hline \multirow[t]{2}{*}{$\mathrm{BMI}\left(\mathrm{kg} / \mathrm{m}^{2}\right)$} & Median (IQR) & $30.3(24.1-38.2)$ & 34.3) & 0.376 \\
\hline & Obesity & $12(30 \%)$ & $10(22.7 \%)$ & 0.469 \\
\hline \multirow{5}{*}{ 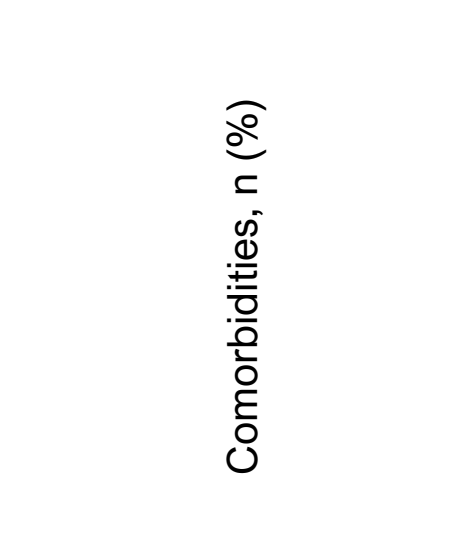 } & Diabetes & $17(42.5 \%)$ & $21(47.7 \%)$ & 0.666 \\
\hline & HTN & 21 (52.5\%) & 27 (61.4\%) & 0.509 \\
\hline & $\mathrm{CHF}$ & $7(17.5 \%)$ & 7 (15.9\%) & $>0.999$ \\
\hline & CAD & $1(2.5 \%)$ & $9(20.5 \%)$ & 0.016 \\
\hline & HIV & $2(5 \%)$ & $3(6.8 \%)$ & $>0.999$ \\
\hline
\end{tabular}




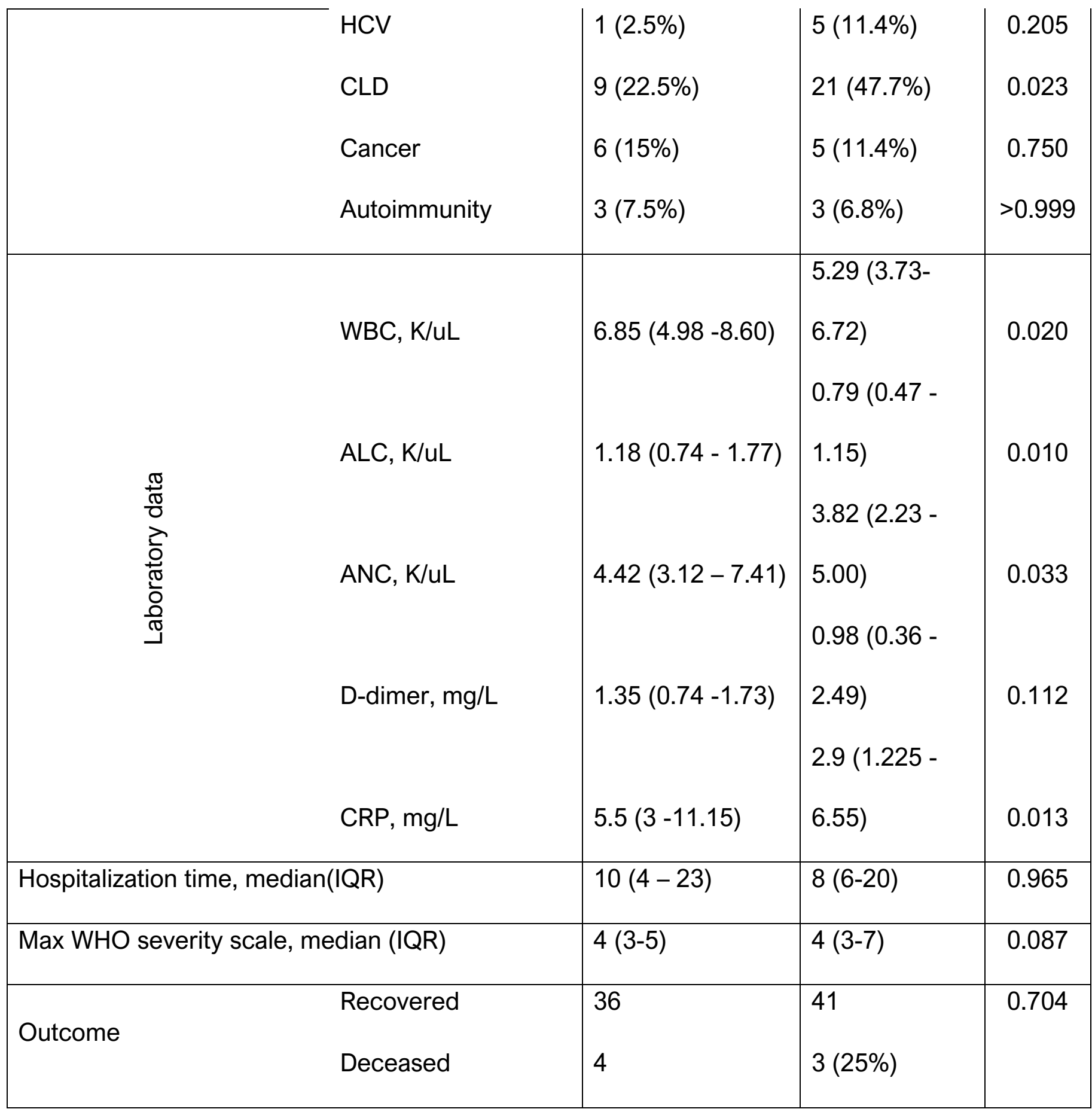


13 . Supplementary Figures 
1 Supplementary Fig. 1. Fragment size distribution of cfDNA in representative samples. A

2 prominent peak length around $\sim 167$ bp indicates a good quality of cfDNA. 

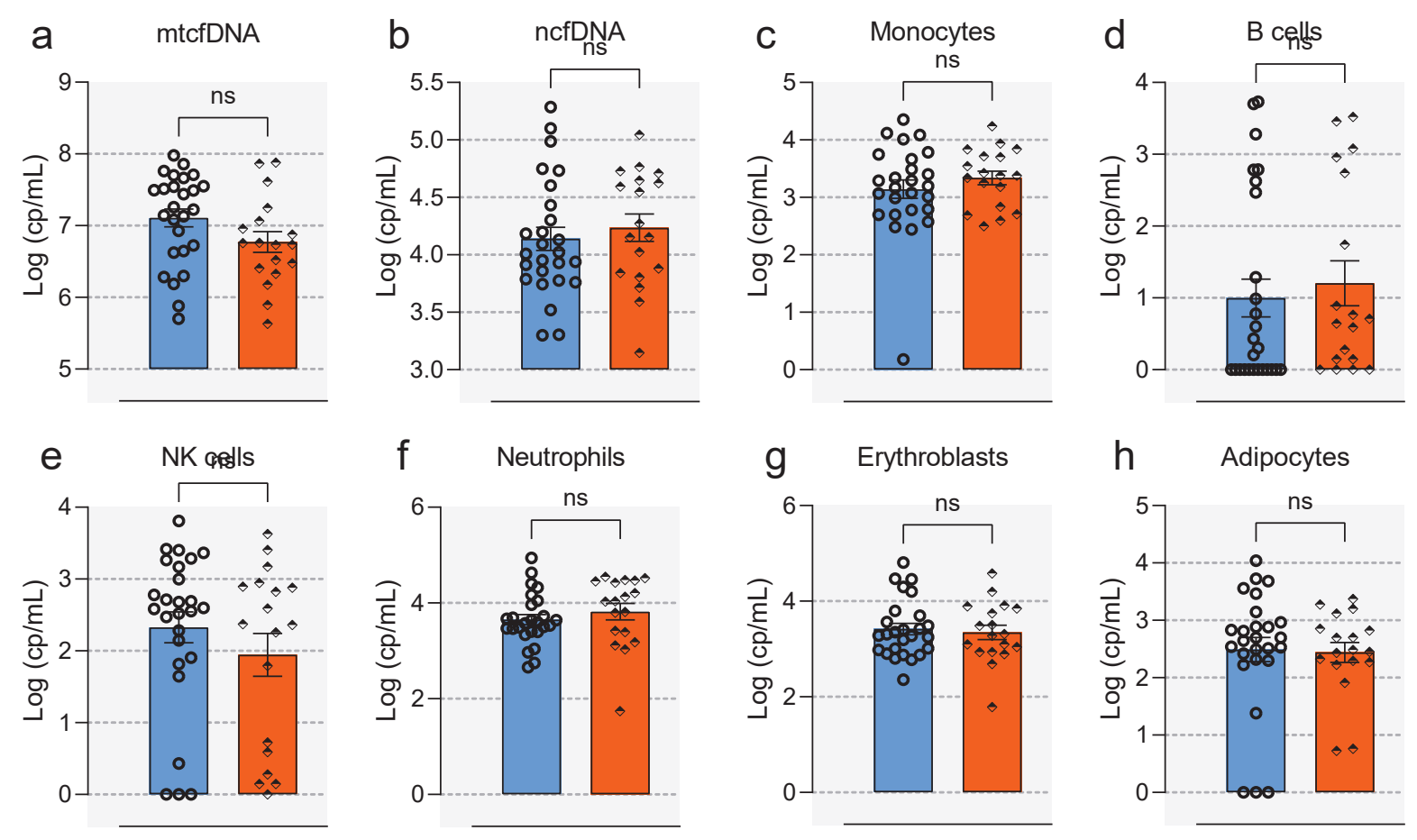

h Adipocytes
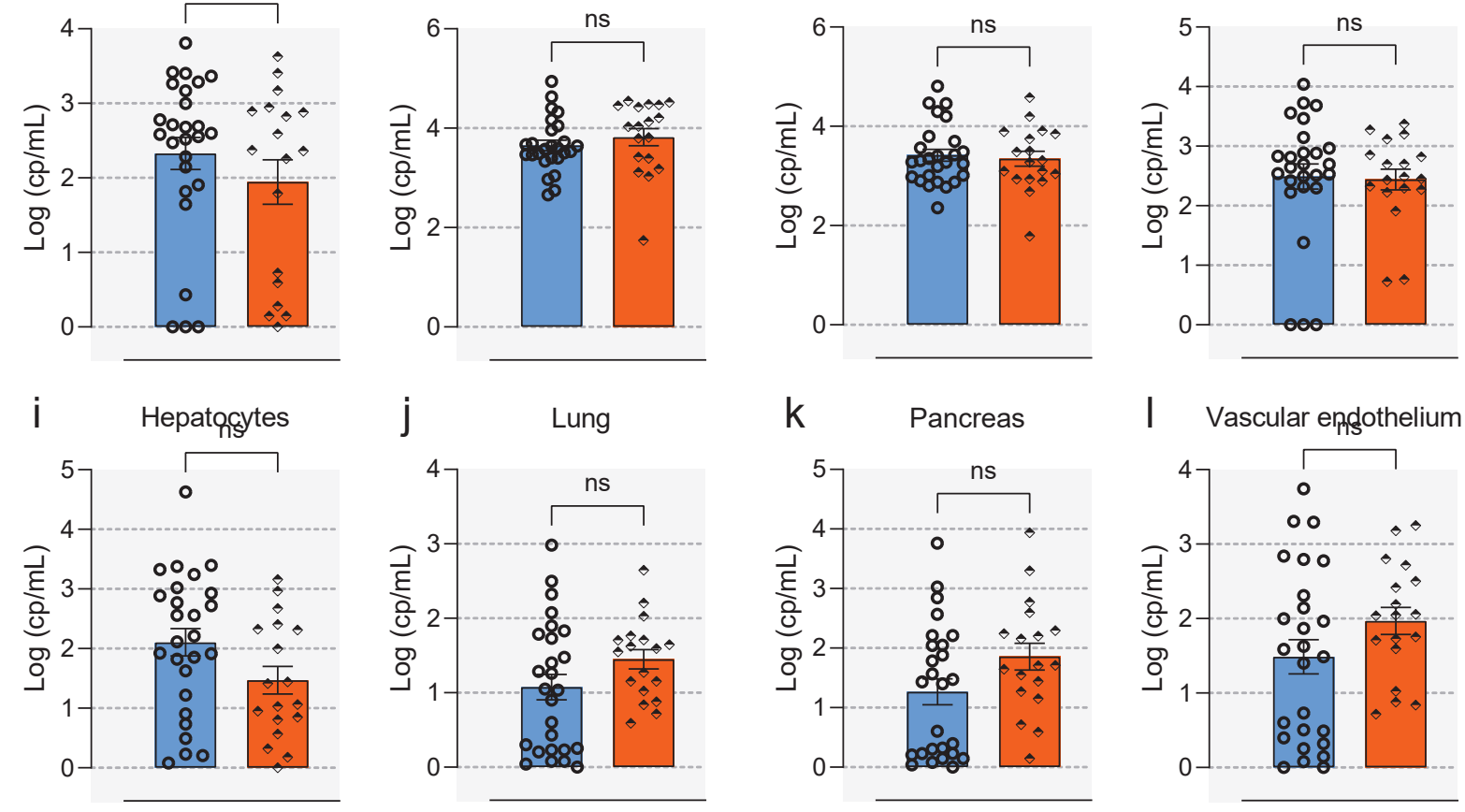

Groups

Other SOT COVOD-19

LTR COVID-19
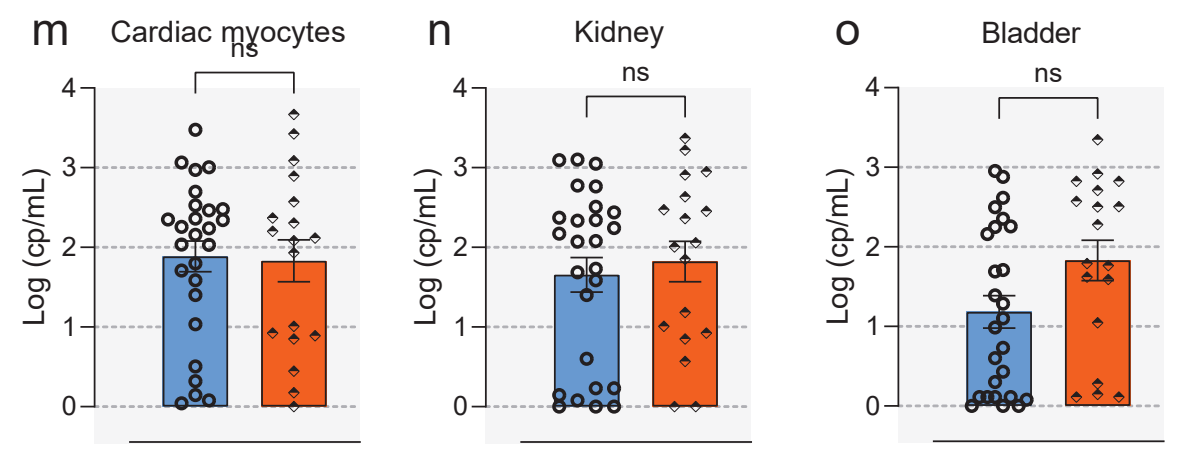

I Vascular endothelium

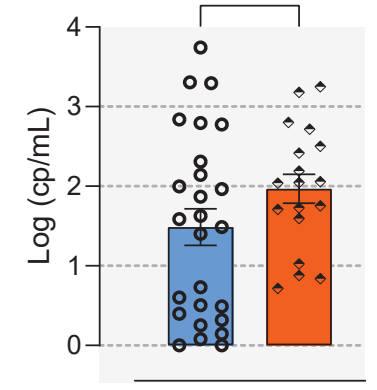

p Squamous epithelium

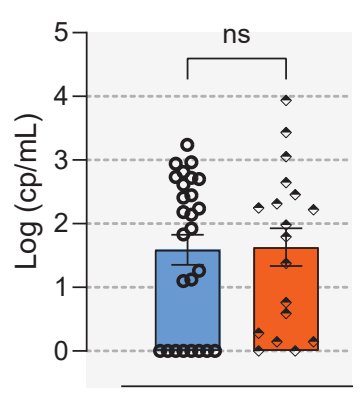


1 Supplementary Fig. 2. Similar cfDNA tissue injury pattern among SOT types.

2 Comparisons of absolute total mtcfDNA (a) ncfDNA and mtcfDNA (b) and tissue-specific

3 cfDNA levels derived monocytes (c), B cells (d), NK cells (e), neutrophils (f), erythroblasts

4 (g), vascular endothelium (h), adipocytes (i), hepatocytes (j), lung (k), pancreas (I),

5 cardiac myocytes $(\mathbf{m})$, kidney $(\mathbf{n})$, bladder $(\mathbf{0})$, and squamous epithelium ( $\mathbf{p}$ ) between lung

6 transplant recipients with COVID-19 $(n=18)$ other SOT types $(n=26)$. Statistical

7 significance was determined by the Mann-Whitney test. Adjusted $p$ values are reported

8 (for multiple comparison and demographic factors (age, sex, and BMI). A p-value $\leq 0.05$

9 and FDR $\leq 0.25$ was considered statistically significant;: FDR $\leq 0.25$ and $p$-value $\leq 0.05,{ }^{* *}$ :

10 FDR $\leq 0.1$ and $p$-value $\leq 0.05,{ }^{* * *}$ : FDR $\leq 0.05$ and $p$-value $\leq 0.05$, NS: FDR $>0.25$ or $p-$ 11 value $>0.05$ 
SOT COVID-19

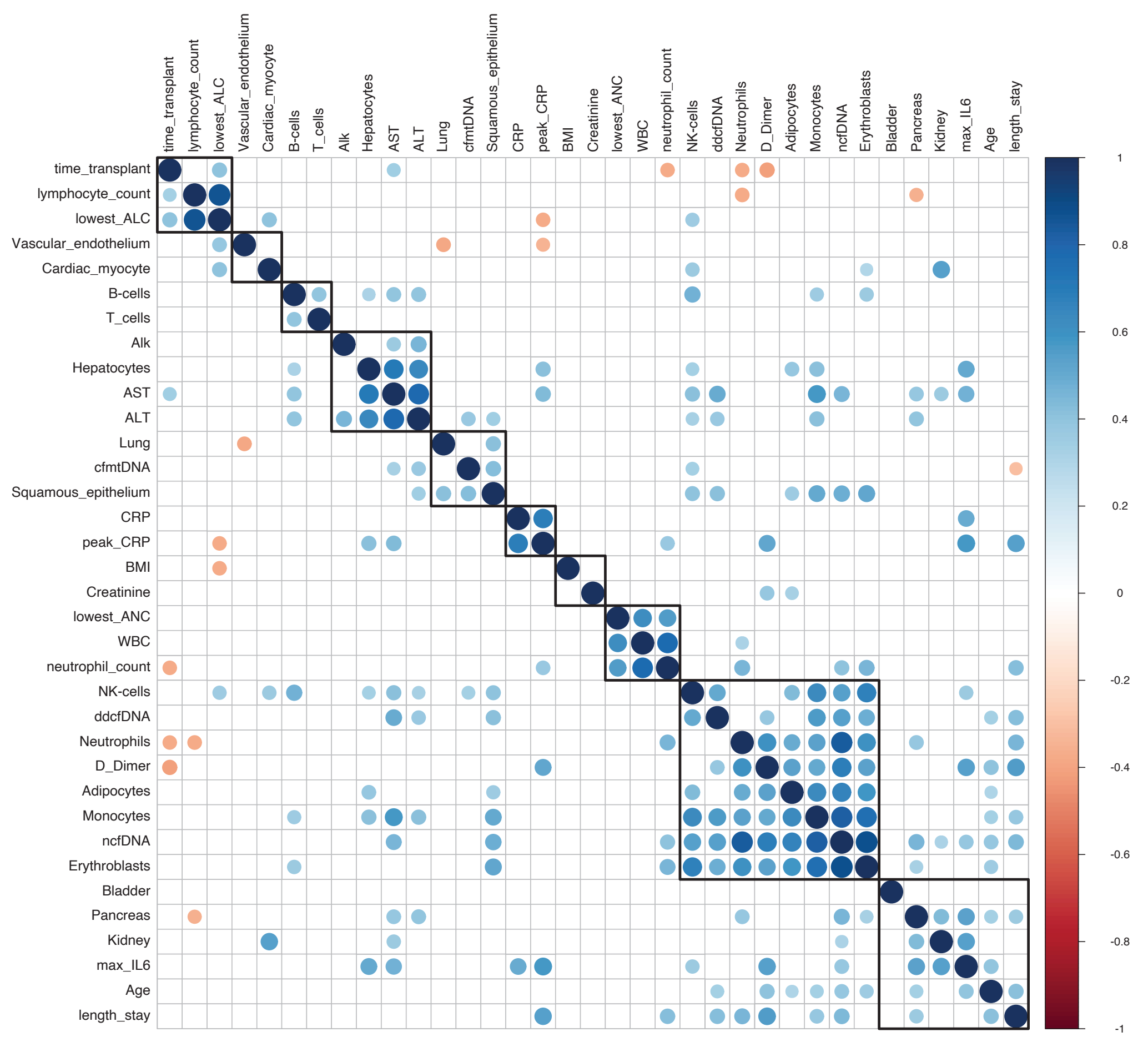


1 Supplementary Fig. 3. Circulating cfDNA levels associated with conventional clinical and

2 biochemical profiles. Heat map between cfDNA and continuous conventional clinical and

3 biochemical profiles conducted upon a Spearman correlation in SOTRs with COVID-19.

4 A $p$-value $\leq 0.05$ and FDR $\leq 0.25$ was considered significant;: FDR $\leq 0.25$ and $p$-value $\leq$

$50.05,{ }^{* *}$ : FDR $\leq 0.1$ and $p$-value $\leq 0.05,{ }^{* *}$ : FDR $\leq 0.05$ and $p$-value $\leq 0.05$, NS: FDR $>0.25$

6 or p-value $>0.05$. 


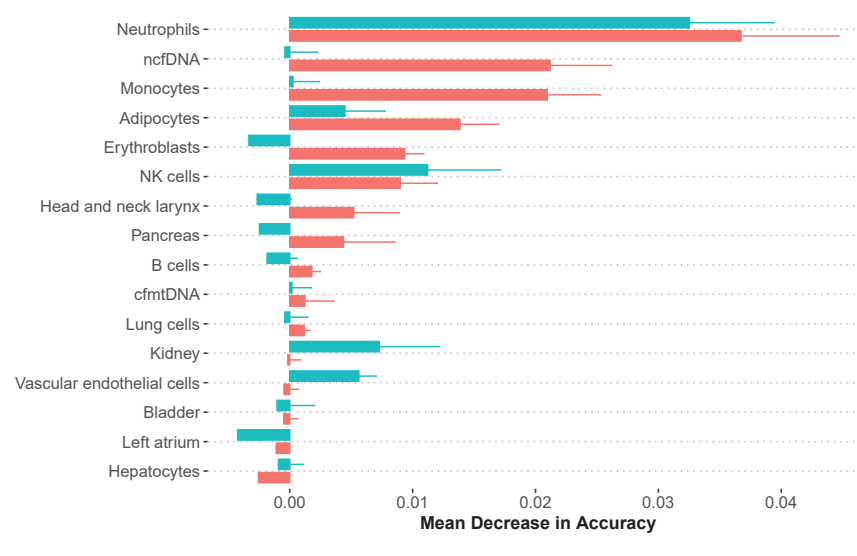

c

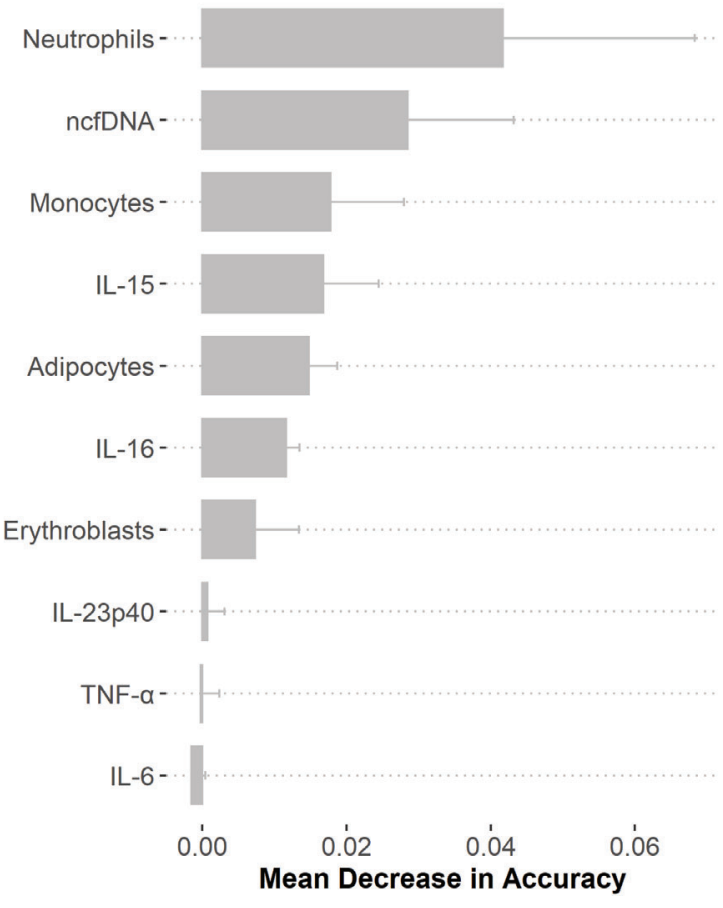

b

IL-15-

IL-16-

TNF- $\alpha-$

IL-23p40 -

IL-6 -

IL-1 $\alpha$ -

IL-8-

MIP-1 $\alpha-$

MCP-1 -

GM-CSF -

IL-5-

IL-1RA-

IL-2Ra-

MIP- $1 \beta-$

MCP-4 -

IFN- $\beta$ -

TNF- $\beta$ -

VEGF -

IFN- $\lambda 1$ -

IL-12p70-

TARC-

IL-2-

IL-1 $\beta$ -

IFN-Y-

MDC-

IFN- $\alpha 2 \mathrm{a}-$

IL-10-

IL-18

IL-17A -

IL-13-

Eotaxin-

Eotaxin -
IL-4 -

IP-10-

Eotaxin-3-

IL-7 -

MCP-2-

100
SOT Non-SOT

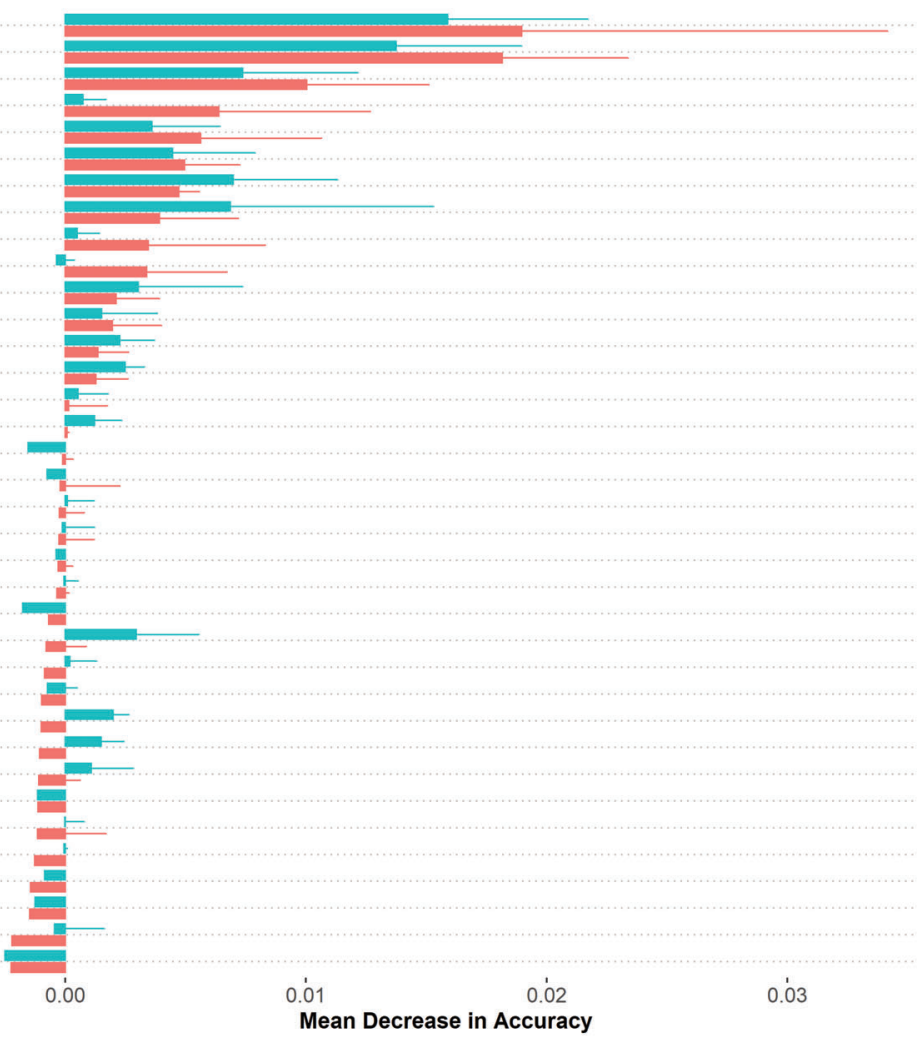

d




1 Supplementary Fig. 4. Plasma cfDNA and cytokine signatures identify patients with

2 severe disease. (a) Rank of important cfDNA features to identify patients with severe

3 disease in SOT and Non-SOT COVID-19 patients based on random forest model (b) Rank

4 of important cytokines features to identify patients with severe disease in SOT and Non-

5 SOT COVID-19 patients based on random forest model. (c) Top five cfDNA and cytokine

6 features to identify SOT patients with severe disease. (d) Performance of top five cfDNA

7 and cytokine features to identify severe SOT patients from mild/moderate groups. 


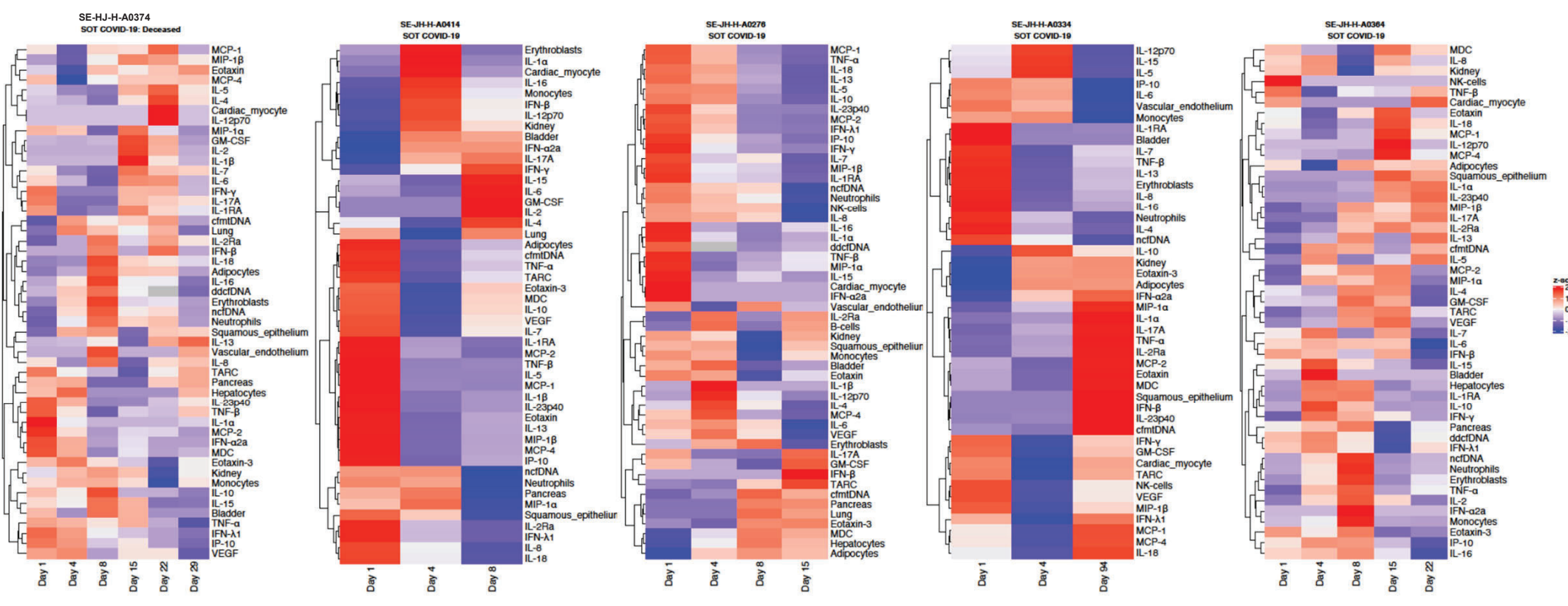


1 Supplementary Fig. 5. Heatmap of longitudinal cfDNA level and cytokine response of 2 prototype SOT COVID-19 patients. Changes in cfDNA measures and cytokine levels over

3 time for SOT patients with severe disease who died (Deceased); with unresolved tissue

4 injury and cytokine pattern (a) and a gradually decreasing cfDNA and cytokine levels in 5 SOTRs who recovered (b-e). 
1 Supplementary Fig. 6. Association between cfDNA and cytokine profiles in SOT COVID-

219 patients subgroups based on disease severity. Pearson correlation matrix between

3 cfDNA and cytokines/chemokines profiles in SOTRs with mild/moderate disease $(n=32)$

4 (a) and severe disease $(n=12)(b)$. Hierarchical Clustering Heatmap of circulating cfDNA

5 and cytokine profiles in SOT patients with Mild/moderate (c) and severe COVID-19

6 disease (d). Spearman correlations test and linear regression analysis were performed

7 to determine statistical significance. A p-value $\leq 0.05$ and FDR $\leq 0.25$ was considered

8 significant; $*$ FDR $\leq 0.25$ and $p$-value $\leq 0.05$, **: FDR $\leq 0.1$ and $p$-value $\leq 0.05$, ${ }^{* * *}$ : FDR $\leq$

90.05 and $p$-value $\leq 0.05$, NS: FDR $>0.25$ or $p$-value $>0.05$. Adjusted $p$ values are reported

10 (for multiple comparison and demographic factors (age, sex, and BMI). 


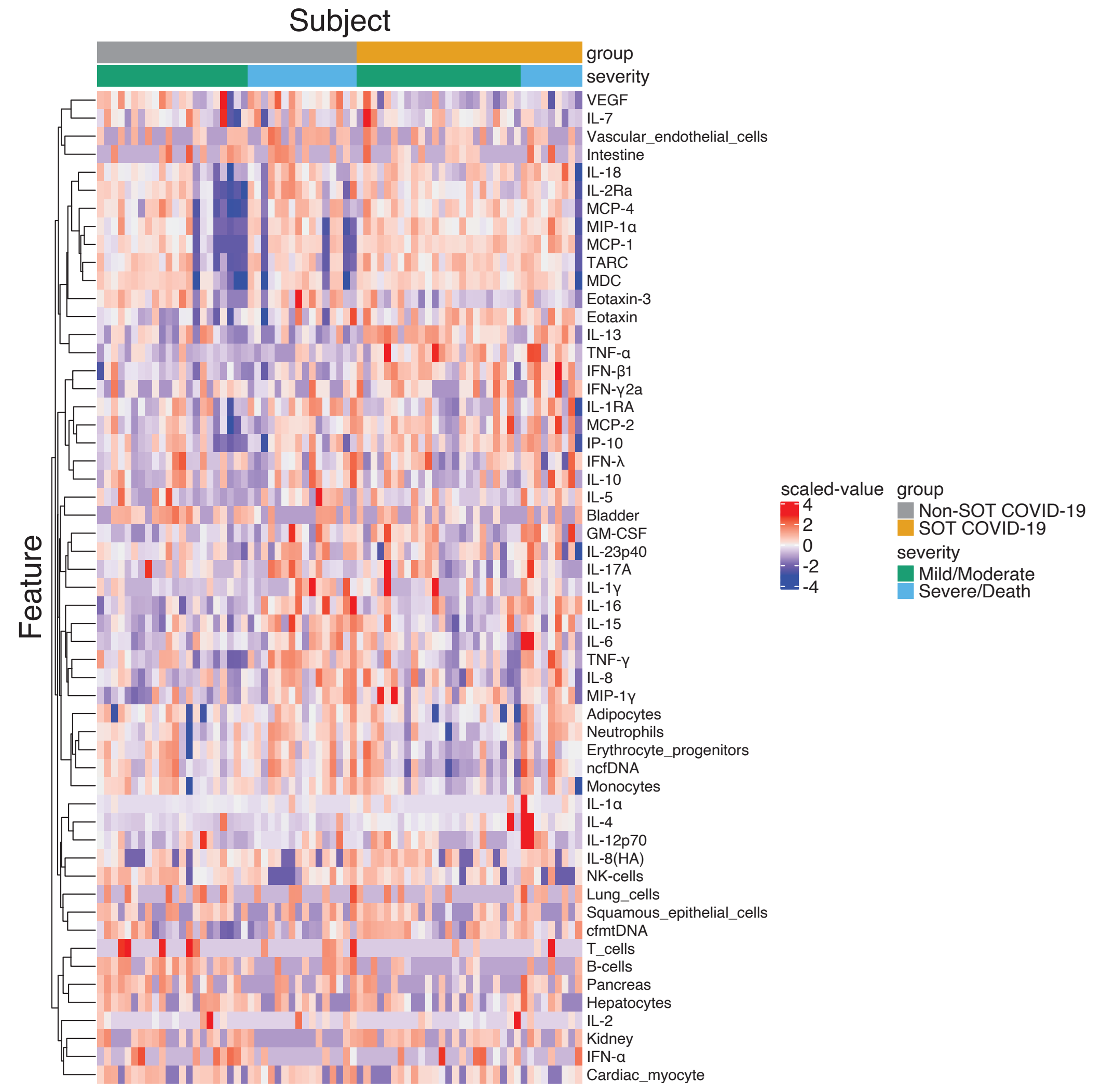


1 Supplementary Fig. 7. Heatmap showing divergent tissue injury patterns and cytokine 2 response that correlated with disease severity in SOT COVID-19 patients. 
1 Supplementary Fig. 8. t-statistics scatterplot plots between cfDNA and cytokine levels

2 in SOT patients with COVID-19. Linear regression analysis between cfDNA features and 3 cytokine profile was conducted. 

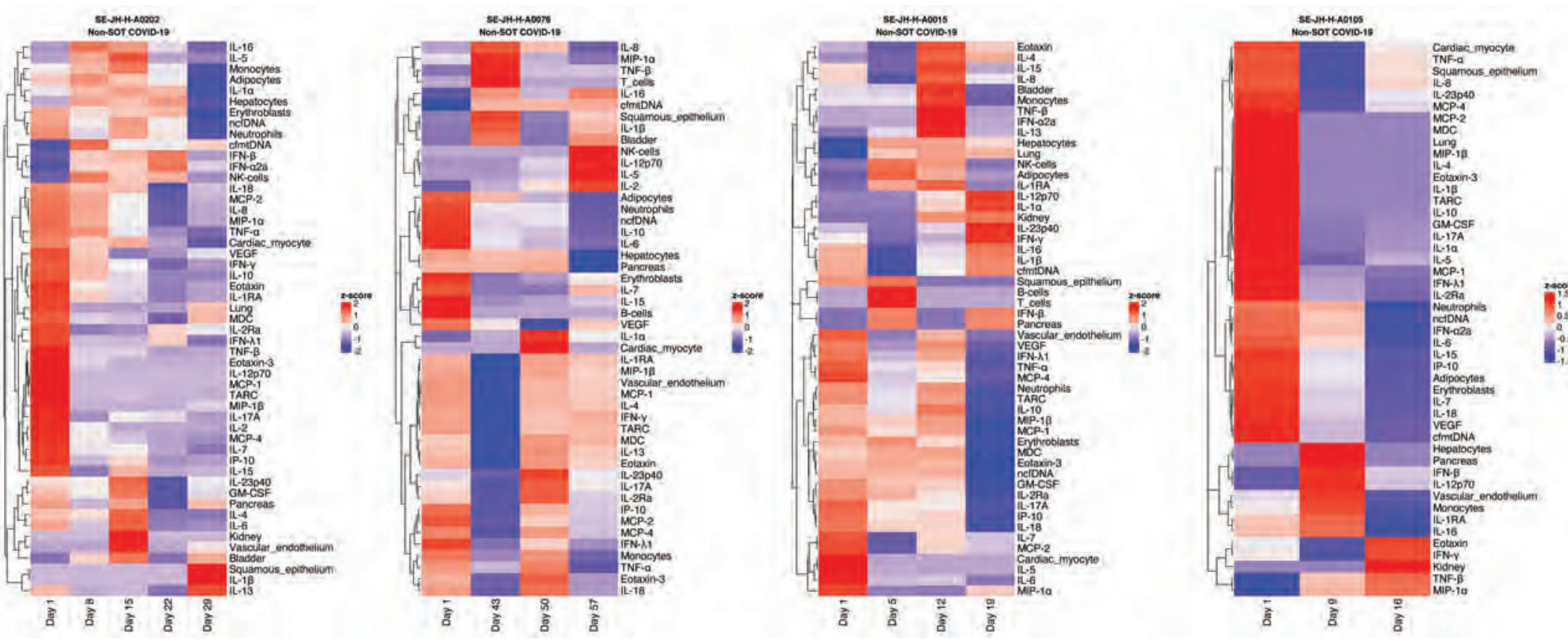
2 Supplementary Fig. 9. Heatmap of longitudinal cfDNA level and cytokine response of 3 prototype Non-SOT COVID-19 patients. 